MAURICIO MICHALAK SENDESKI

\title{
Atividade simpática na hipertensão arterial associada à compressão neurovascular do bulbo rostral ventro-lateral em humanos
}

Tese apresentada à Faculdade de Medicina da Universidade de São Paulo para obtenção do título de Doutor em Ciências.

Área de concentração: Fisiopatologia Experimental

Orientador: Prof. Dr. Eduardo Moacyr Krieger

São Paulo 
SUMÁRIO

Resumo

Summary

1. INTRODUÇÃO

1.1. Associação entre hipertensão arterial sistêmica e compressão neurovascular 1

1.2. Mecanismos de regulação da pressão arterial no Tronco Cerebral 4

1.3. Modelos experimentais de compressão neurovascular no bulbo 6

1.4. Tratamento cirúrgico da compressão neurovascular em hipertensos $\quad 7$

1.5. Atividade simpática, hipertensão arterial e compressão neurovascular $\quad 8$

1.6. Objetivos 11

2. MÉTODOS 12

$\begin{array}{lr}\text { 2.1. Casuística } & 12\end{array}$

2.2. Avaliação clínica 13

2.3. Exame de Ressonância Magnética 14

2.4. Avaliação de Atividade Simpática 15

2.4.1. Registro dos sinais 15

$\begin{array}{ll}\text { 2.4.2. Seqüência experimental } & 17\end{array}$

2.4.3. Análise dos dados 19

2.5. Análise estatística 20

3. RESULTADOS 22

3.1. Exames de Ressonância Magnética 22

3.2. Avaliação clínica $\quad 27$

3.3. Avaliação de Atividade Simpática 29

3.3.1. Período basal $\quad 30$ 
3.3.3. Resposta ao exercício isométrico

3.4. Sumário dos resultados

4. DISCUSSÃO

5. CONCLUSÕES

6. ANEXOS 54

7. REFERÊNCIAS BIBLIOGRÁFICAS 
SENDESKI, MM. Avaliação da atividade simpática na hipertensão arterial associada à compressão neurovascular. São Paulo, 2004. 85p. Tese (Doutorado) - Faculdade de Medicina, Universidade de São Paulo.

Foi investigado, utilizando imagem por ressonância magnética (IMR), a prevalência de sinais de compressão neurovascular (CNV) no bulbo rostral ventro-lateral (RVL) de 64 indivíduos com hipertensão arterial primária $(150 \pm 13 \mathrm{mmHg})$ e de 29 voluntários normotensos $(120 \pm 14 \mathrm{mmHg})$. Pesquisou também a atividade simpática do nervo muscular (ASNM) em 25 dos hipertensos, correlacionando-a com o resultado da IMR. Foram excluídos do estudo indivíduos com evidência de doença vascular e com condições clínicas capazes de alterar a atividade simpática. Hipertensos e normotensos foram separados, segundo o aspecto da IRM, em três grupos: $\mathrm{CNV}-$, sem sinais de CNV na IRM (34\% dos hipertensos e $73 \%$ dos normotensos); CNV+ Contato Vascular, com imagem vascular junto ao bulbo RVL sem causar deformação anatômica (39\% dos hipertensos e $24 \%$ dos normotensos); e CNV+ Compressão Vascular, com imagem vascular causando franca compressão no bulbo RVL ( $27 \%$ dos hipertensos e $3 \%$ dos normotensos). As diferenças entre os grupos foram estatisticamente significantes. Em 25 hipertensos a ASNM foi estudada por microneurografia do nervo fibular, em repouso e em resposta aos estímulos hipotérmico (teste do gelo) e exercício isométrico. A freqüência cardíaca e a pressão arterial batimento a batimento foram monitoradas continuamente durante o teste. Nove indivíduos eram do grupo CNV-, 8 do grupo CNV+ Contato Vascular, e 8 do grupo $\mathrm{CNV}+$ Compressão Vascular. $\mathrm{O}$ grupo $\mathrm{CNV}+$ Compressão Vascular apresentou, durante o período basal, ASNM significativamente mais elevada que os grupos CNV+ Contato Vascular e CNV- $(30,4 \pm 3,4$ vs. 17,5 $\pm 1,1$ e 21,4 $\pm 3,2$, em espículas, respectivamente). A resposta da ASNM ao gelo e ao exercício isométrico e 
as medidas de pressão arterial e freqüência cardíaca basais e após estímulos não foram diferentes entre os grupos. Esses resultados mostram que existe uma a associação entre a presença de sinais de CNV na IRM e a HAS, e que o aspecto de franca compressão vascular $(\mathrm{CNV}+$ Compressão) é praticamente exclusivo de hipertensos. Mostram, também, ASNM elevada no grupo com achado radiológico sugestivo de $\mathrm{CNV}$ do tipo $\mathrm{CNV}+$ Compressão, enquanto o grupo $\mathrm{CNV}+$ Contato Vascular teve ASNM semelhante ao grupo CNV-. Essas evidências são importantes para a identificação de subgrupos de hipertensos cuja imagem de CNV na IRM tenha implicação fisiopatológica para a hipertensão. 


\begin{abstract}
SENDESKI, MM. Sympathetic activity in hypertension related to neurovascular compression. São Paulo, 2004. 85p. Tese (Doutorado) - Faculdade de Medicina, Universidade de São Paulo.
\end{abstract}

This study used magnetic resonance imaging (MRI) to investigate the prevalence of neurovascular compression (NVC) at the rostral ventro-lateral medulla in 64 subjects with arterial hypertension $(150 \pm 13 \mathrm{mmHg})$ and in 29 normotensive volunteers (120 $\pm 14 \mathrm{mmHg}$ ). The muscle nerve sympathetic activity (MNSA) was measured in 25 of the subjects with hypertension, and the results were related to MRI. Subjects with vascular disease and conditions that could alter sympathetic activity were not included in the study. Both normotensive volunteers and subjects with hypertension were included, according to MRI result, in one of three groups: NVC-, without signs of NVC at MRI (34\% of hypertension group and $73 \%$ of normotensive); NVC+ Vascular Contact, with the image of an artery in contact with RVL medulla, but not compressing it (39\% of hypertension group and $24 \%$ of normotensive); and NVC+ Vascular Compression, with the image of an artery causing compression on RVL medulla (27\% of hypertension group and $3 \%$ of normotensive). The differences among groups were statistically significant. Microneurography of fibular nerve was used to measure MNSA in 25 subjects of hypertension group, during rest conditions and after hypothermic stimulus and isometric exercise. Heart rate and blood pressure were continuously recorded (beat-to-beat) during the test. Nine out of the 25 subjects had NVC- aspect at MRI, 8 had NVC+ Vascular Contact aspect, and 8 had NVC+ Vascular Compression. MNSA was significantly higher during rest in $\mathrm{NVC}+$ Vascular Compression group $(30,4 \pm 3,4$ vs. $17,5 \pm 1,1$ e 21,4 $\pm 3,2$ spikes, 
respectively), but did not differ in response to stimuli. Heart rate and blood pressure did not differ among groups during rest and after hypothermic and isometric exercise stimuli. Our results show an association between arterial hypertension and the detection of NVC at the RVL medulla on MRI, especially because and the aspect of frank compression (NVC+ Vascular Compression type) is found almost exclusively in subjects with hypertension. We also found that MNSA is higher in the NVC+ Vascular Compression group, while NVC+ Vascular Contact has MNSA similar to NVC- group. These facts are important to identify hypertensive patients with NVC that may have pathophysiological importance. 


\section{INTRODUÇÃO}

É bem conhecida a alta prevalência da Hipertensão Arterial Sistêmica (HAS) no homem e sua relação com as doenças cardiovasculares, bem como a importância destas ao aparecerem atualmente como a principal causa de mortalidade não traumática em países desenvolvidos ou em desenvolvimento. Na maioria dos casos de HAS não é possível descobrir uma causa única, sendo denominada nesta situação HAS primária, dependente de múltiplos fatores (multifatorial) e vários genes (poligênica). Todavia, são conhecidas inúmeras situações clínicas associadas à HAS primária, como história familiar, obesidade, inatividade, origem étnica, dentre outras, que imputam um comportamento clínico peculiar ao indivíduo que as apresenta, e que se consideradas podem trazer benefícios terapêuticos ou medidas preventivas de caráter individual (LOTUFO, 1998; HADDAD et al., 2000; HANSSON et al., 2000; RUIZ et al., 2001). A Compressão Neurovascular (CNV), um achado neuroanatômico anômalo da região do tronco cerebral, tem sido descrita em associação com a HAS primária. No entanto, pouco se sabe sobre as características clínicas específicas dos indivíduos portadores desse achado, e nosso trabalho tem como objetivo contribuir para um melhor entendimento desta entidade.

\subsection{Associação entre hipertensão arterial sistêmica e compressão neurovascular}

O conceito de compressão de uma estrutura neural por um vaso sangüíneo como fator etiológico de disfunções neurológicas (compressão neurovascular) foi desenvolvido por Dandy, e aprimorado por Gardner e Jannetta (JANNETTA, 1980; WILKINS, 1985). É reconhecida sua relação com entidades como neuralgia do trigêmeo, espasmo hemifacial e neuralgia do glossofaríngeo. Quando o tratamento 
clínico destas entidades não tem sucesso faz-se indicação de tratamento cirúrgico por descompressão neurovascular (afastando o vaso que comprime a estrutura neural, e interpondo material inerte para impedir o seu retorno à posição).

A descrição inicial de associação entre CNV e hipertensão arterial em humanos foi feita por Jannetta, em 1979, em indivíduos submetidos à descompressão neurovascular para tratamento de entidades como neuralgias e disfunções de nervos cranianos. Observou haver associação de hipertensão arterial primária com o achado intra-operatório de $\mathrm{CNV}$ no bulbo rostral ventro-lateral (RVL) à esquerda. Posteriormente, o mesmo autor descreveu a evolução de indivíduos que, sendo hipertensos e submetidos à descompressão neurovascular por outros motivos, foram tratados no mesmo ato cirúrgico com descompressão neurovascular do bulbo RVL (JANNETTA et al., 1979; JANNETTA, 1980). De 42 indivíduos, 32 apresentaram melhora inicial significativa nos valores de pressão arterial (JANNETTA et al., 1985a). Na maioria dos casos o vaso anômalo encontrado foi a artéria cerebelar posterior inferior.

Após os relatos iniciais de Jannetta, baseados em observação intra-operatória de CNV no bulbo, outros métodos foram utilizados para verificar a associação entre hipertensão arterial e CNV de bulbo RVL em humanos. Kleineberg, em 1991, publicou uma série na qual utilizou angiografias cerebrais para detecção de sinais de CNV no bulbo RVL. Encontrou que em 99 hipertensos, 81\% tinham indícios de CNV, e em 57 normotensos, 41.7\% apresentavam indícios de CNV. Em 1992 uma ampliação da mesma série mostrou resultados semelhantes (KLEINEBERG et al., 1991; KLEINEBERG et al., 1992). 
Em 1992 Naraghi relatou em uma série de 55 indivíduos (24 com hipertensão primária, 10 com hipertensão secundária e 21 normotensos) a dissecção microanatômica do bulbo durante necropsia. Havia CNV no bulbo ventrolateral em todos os indivíduos com hipertensão arterial primária e em dois pacientes normotensos, mas em nenhum com hipertensão secundária. Não houve diferença histopatológica entre os grupos. Foram descritos ainda três padrões de CNV do bulbo: compressão pela artéria cerebelar posterior inferior (mais comum), pela artéria vertebral ou por ambas (NARAGHI et al., 1992).

A utilização da ressonância magnética $(\mathrm{RM})$ para verificação de sinais de CNV associada à hipertensão (NARAGHI et al., 1994) demonstrou ser este método capaz de detectar alterações na anatomia da região de forma não invasiva. Vários estudos em grupos com tempo de exposição à hipertensão e faixa etária semelhantes mostraram freqüências de 74 a $90 \%$ de sinais de CNV nos pacientes com hipertensão arterial primária, de 7 a $22.2 \%$ nos normotensos, e de 11 a $16.6 \%$ no grupo com hipertensão secundária (NARAGHI et al., 1994; AKIMURA et al., 1995; PIERPAOLO et al., 1996; MORIMOTO et al., 1997). Esses resultados são semelhantes aos números encontrados por Naraghi utilizando dissecção microanatômica (NARAGHI et al., 1994). Mostram também que a alteração na posição vascular encontrada provavelmente não é resultado da exposição crônica a níveis pressóricos elevados, visto que os pacientes com hipertensão secundária tiveram freqüência de CNV semelhante à dos normotensos.

Algumas séries utilizando IRM para investigação de CNV não demonstraram diferença entre hipertensos e normotensos (WATTERS et al., 1996; COLON et al., 1998; SAGLITZ et al., 2002). Nesses estudos, no entanto, houve viés tanto na 
seleção dos indivíduos como na técnica utilizada na aquisição das imagens de ressonância. Não foi descrito, por exemplo, o tempo de diagnóstico da hipertensão, os grupos não eram pareados quanto à idade, não foram descritos os níveis de hipertensão arterial e o tratamento, não foram estudados casos de hipertensão secundária, e, na série de Watters (WATTERS et al., 1996), a aquisição de IRM não foi realizada com técnica apropriada à detecção de CNV.

\subsection{Mecanismos de regulação da pressão arterial no Tronco Cerebral}

O conhecimento da importância do bulbo no controle da pressão arterial remonta aos estudos de Dittmar, em 1873, realizando transecções em SNC de coelhos. Até a década de 60, entretanto, não era possível delimitar áreas específicas do bulbo relacionadas ao controle pressórico, pois o método até então utilizado (estimulação elétrica) estimulava também fibras de passagem próximas ao ponto de estimulação, mascarando os resultados. Somente a partir de 1972, com os trabalhos de Guertzenstein, foi possível discriminar as regiões do bulbo envolvidas no controle pressórico (GUERTZENSTEIN, 1972; GUERTZENSTEIN, 1973). Utilizando aplicação tópica (e posteriormente injeções) de substâncias inibitórias e excitatórias de ação seletiva pós-sináptica no bulbo de gatos, foi possível identificar a topografia de dois grupos celulares distintos: 1) a área rostral ventrolateral (RVL), com efeitos pressores quando estimulada e depressores quando inibida e 2) a área caudal ventrolateral, com efeitos contrários (HOROWITZ, 1999; WYSS et al., 1999). 


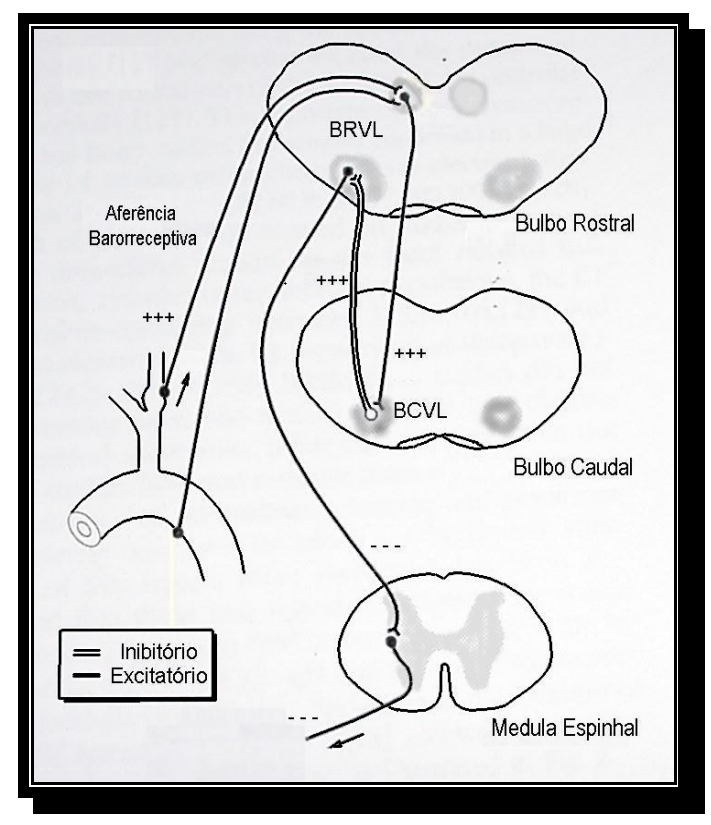

Figura 1 - Representação da integração autonômica simpática no bulbo (CHALMERS et al., 1991).

Grupos de neurônios da área RVL possuem um padrão de atividade rítmica intrínseca (automaticidade), que é continuamente influenciada por aferências dos baroreceptores e de outras regiões do SNC. Dessa forma, parecem funcionar como um marcapasso da atividade simpática no SNC (BRODY et al., 1991; WYSS et al., 1999). Quando associados à constatação de que dois terços das eferências dos neurônios produtores de adrenalina da área RVL dirigem-se para a medula torácica (coluna intermédio-lateral), tais dados sugerem que a área RVL tenha papel importante numa via final eferente simpática, capaz de influenciar o controle cardiovascular (BELL, 1990; KIELY et al., 1994). O papel pressor da área RVL foi também verificado em várias outras circunstâncias: as respostas pressoras observadas após desnervação sinoaórtica, destruição dos núcleos do trato solitário, estímulo do núcleo 
fastigial e obscurus da rafe, e estímulo dos nervos ciáticos, são significativamente atenuadas após destruição ou inibição da área RVL (OPARIL et al., 1990;

CHALMERS et al., 1991; CAMPOS et al., 1997).

\subsection{Modelos Experimentais de Compressão Neurovascular no Bulbo}

A possibilidade de que a compressão neurovascular do bulbo RVL possa estar associada a estados de hipertensão arterial, como sugeriram Jannetta e Gendell (JANNETTA et al., 1979), estimulou a pesquisa em animais. Em 1985 Jannetta (JANNETTA et al., 1985b) descreveu um modelo de hipertensão arterial em babuínos provocado pela compressão pulsátil do bulbo rostral ventrolateral, utilizando balões com pulsatilidade transmitida a partir da aorta do animal. Havia aumentos na pressão arterial e no débito cardíaco, associados a leve bradicardia. As alterações eram mantidas por períodos de até quatro semanas de insuflação do balão, regredindo após esvaziamento.

Em 1997 Morimoto (MORIMOTO et al., 1997) descreveu um modelo de compressão pulsátil em ratos Wistar, com balão próximo ao bulbo RVL, obtendo resultados semelhantes aos de Jannetta em babuínos. Também foi feita a monitoração de atividade simpática do nervo esplâncnico, encontrando-se um aumento tanto da pressão arterial como da frequiência cardíaca e da atividade nervosa simpática. $\mathrm{O}$ aumento da atividade simpática foi interpretado como responsável pela resposta pressora associada à compressão pulsátil do bulbo.

Em 2000, em modelo experimental semelhante, Morimoto observou a primeira evidência direta de alteração neuroquímica em neurônios do bulbo RVL em animais com compressão experimental: a resposta pressora à compressão do bulbo 
RVL com balão foi significantemente inibida após microinjeção local de antagonistas de glutamato em ratos. Estes dados sugerem que a compressão pulsátil do bulbo RVL leva a uma hiperatividade glutamatérgica local, associada à hiperatividade simpática já verificada neste modelo (MORIMOTO et al., 2000).

\subsection{Tratamento cirúrgico da compressão neurovascular em hipertensos}

A descompressão neurovascular do bulbo em indivíduos hipertensos essenciais durante cirurgia motivada por outra patologia produziu queda duradoura da pressão arterial em alguns indivíduos (JANNETTA et al., 1985a; PLATANIA et al., 1997). Este fato influenciou que pacientes com hipertensão primária fossem submetidos à descompressão neurovascular especificamente para tratamento da hipertensão arterial (GEIGER et al., 1998; LEVY et al., 1998).

Em 1998 Levy publicou os resultados de 11 indivíduos com hipertensão arterial primária refratária (7 com disautonomia associada) e um indivíduo com disautonomia severa, todos com $\mathrm{CNV}$ de bulbo RVL na RM, tratados com descompressão neurovascular. Oito indivíduos evoluíram com redução maior que 20mmHg na pressão arterial, e cinco com melhora da disautonomia, durante seguimento médio de 49.5 meses (LEVY et al., 1998).

Em 1998 Geiger descreveu uma série de 8 indivíduos com hipertensão refratária e CNV no bulbo RVL demonstrada na RM, submetidos a cirurgia para descompressão neurovascular. Foi obtido controle da hipertensão a longo prazo em 4 e melhora dos níveis pressóricos em 3, persistindo o uso de medicação, conforme relato em publicação posterior (GEIGER et al., 1998; FRANK et al., 2001). 


\subsection{Atividade simpática, hipertensão arterial e compressão neurovascular}

A avaliação da atividade simpática em humanos pode ser aferida utilizandose várias metodologias, como atividade simpática no nervo fibular, variabilidade de frequiência cardíaca, "spillover" de noradrenalina e bloqueios farmacológicos. A atividade simpática do nervo muscular (ASNM), por microneurografia, fornece evidências diretas e fidedignas da atividade simpática regional para o território inervado, guardando relação com a atividade simpática global. É o único método atualmente disponível para aferir diretamente, em humanos, a atividade neural de origem autonômica, pois os outros métodos citados são indiretos e baseados na repercussão da atividade autonômica no sistema circulatório (DELIUS et al., 1972; GRASSI, 1998; MANCIA et al., 1998; GRASSI et al., 1999).

Existem evidências claras de hiperatividade simpática em pacientes com hipertensão primária (GRASSI, 1998; JENNINGS, 1998; NOLL et al., 1998; WYSS et al., 1999). No entanto, em hipertensos com CNV a atividade simpática não foi ainda investigada em profundidade. A primeira série publicada foi de Makino (MAKINO et al., 1999), comparando a atividade simpática entre 14 hipertensos primários com e 11 sem CNV na RM. No grupo com CNV os níveis plasmáticos de noradrenalina eram significativamente mais altos, e a sensibilidade baroreflexa, pesquisada com infusão de fenilefrina, estava levemente diminuída, sugerindo atividade simpática aumentada. Não houve diferença entre os grupos nas respostas de pressão arterial e da variabilidade de freqüência cardíaca ao stress mental, frio, "hand grip", manobra de Valsalva, infusão de fenilefrina e teste da clonidina. A monitorização de atividade nervosa simpática muscular não foi utilizada nesta série. 
Morimoto relatou em 1999 (MORIMOTO et al., 1999) o caso de um indivíduo com hipertensão primária submetido a descompressão neurovascular de nervo facial indicada primariamente para espasmos hemifaciais refratários, sendo feita também descompressão da CNV de bulbo RVL, detectada na IRM préoperatória. Houve, após a cirurgia, indícios de diminuição da atividade simpática: diminuição da pressão arterial, queda dos níveis plasmático e urinário de noradrenalina, mudança do padrão de variabilidade da freqüência cardíaca de baixa para alta freqüência, e queda de atividade nervosa simpática muscular à microneurografia.

Morise, em 2000 (MORISE et al., 2000), comparou a variabilidade da frequiência cardíca entre 23 indivíduos com HAS primária, 13 indivíduos com HAS secundária, e 46 normotensos. A "power spectral density" de baixa freqüência (APSD, 0.04 a $0.15 \mathrm{~Hz}$ ) ajustada à idade, um indicador de tônus simpático, estava significativamente mais elevada nos hipertensos primários com $\mathrm{CNV}$ do que naqueles sem CNV. Era maior também do que em normotensos com e sem CNV, e do que em hipertensos secundários. Não houve diferença, entre os grupos, na A-PSD de baixa freqüência $(0.15$ to $0.40 \mathrm{~Hz})$, indicadora de tônus vagal.

Shobel, em 2002 (SCHOBEL et al., 2002), comparou a ASNM de 21 hipertensos com CNV e 12 hipertensos sem CNV, relatando valores basais e em resposta ao estímulo hipotérmico (manobra do gelo) mais elevados no grupo com CNV. A ASNM foi aferida, no entanto, durante uso de medicação anti-hipertensiva, devido à dificuldade prática de se realizar retirada de medicação de forma segura nos indivíduos com níveis pressóricos muito elevados. No estudo não foi considerada a gravidade da hipertensão, mencionando-se apenas que a PA sistólica ambulatorial 
dos grupos era diferente $(158 \pm 28 \mathrm{mmHg}$ no grupo com CNV contra $144 \pm 19$ $\mathrm{mmHg}$ no grupo sem $\mathrm{CNV}$ ). Este fato dificulta a comparação entre os dois grupos, visto que o controle pressórico menos eficiente no grupo com CNV poderia explicar, por si só, a diferença verificada na ASNM. Os grupos com e sem CNV foram divididos, do ponto de vista radiológico, segundo a presença de sinal vascular junto ao bulbo RVL do lado esquerdo somente, e esta descrição parece englobar tanto os achados de simples contato como de franca compressão vascular, embora tal informação não esteja explícitada no texto. São descritos os prováveis vasos envolvidos, mas os dados clínicos, hemodinâmicos e de atividade autonômica são comparados somente entre os grupos com e sem sinais de CNV.

Deduz-se dos dados expostos que uma parcela dos hipertensos considerados primários possui uma anomalia vascular no sistema nervoso central com provável implicação na fisiopatologia de sua hipertensão. Mesmo não sendo o fator primário, poderia ser considerada um fator agravante. O estudo da atividade simpática dos indivíduos com e sem CNV no bulbo RVL é um dos caminhos para o entendimento do mecanismo fisiopatológico pelo qual a CNV determina, ajuda a manter, ou simplesmente se relaciona à hipertensão arterial. É necessário, no entanto, esclarecer melhor a prevalância da CNV na população de hipertensos, identificar com maior precisão o grau de contato e compressão provocado pelas artérias no bulbo RVL e, especialmente, se a CNV no bulbo provoca ou não hiperatividade simpática que pode agravar a hipertensão. 


\subsection{Objetivos}

Os objetivos deste estudo são: 1) determinar a prevalência da CNV na população de hipertensos atendidos pelo Instituto do Coração; 2) dar especial atenção ao aspecto da IRM quanto à presença de contato ou compressão vascular no tecido neural; 3) investigar a atividade simpática em hipertensos sem CNV e com CNV de diferentes intensidades na IRM para avaliar a repercussão clínica dos achados na fisiopatologia da hipertensão. 


\section{MÉTODOS}

\subsection{Casuística}

Participaram do estudo um total de 93 indivíduos, sendo 64 pacientes com Hipertensão Arterial Primária leve, moderada e grave, de ambos os sexos, e idade entre 20 e 55 anos, acompanhados ou iniciando acompanhamento no Ambulatório da Unidade de Hipertensão do InCor, e 29 indivíduos voluntários para composição do grupo de normotensos, de iguais limites etários. Todos os indivíduos foram esclarecidos sobre os objetivos do estudo e assinaram termo de consentimento pósinformado.

Não foram incluídos no protocolo indivíduos com evidência de arteriopatia (insuficiência coronariana, antecedente de infarto do miocárdio, insuficiência arterial periférica crônica, acidente vascular cerebral prévio) e condições clínicas que se relacionem a alterações de atividade simpática (Diabetes Melitus, insuficiência cardíaca congestiva, etc).

Todos os 93 indivíduos (hipertensos e normotensos) foram avaliados clinicamente para confimação do diagnóstico de Hipertensão ou inclusão no grupo de normotensos, e todos foram submetidos a exame de Ressonância Magnética de encéfalo para verificação de presença ou ausência de sinais de CNV no bulbo RVL.

Dentre os indivíduos hipertensos $(\mathrm{N}=64)$, em 25 foi possível avaliar a atividade simpática pela microneurografia. Além do consentimento e colaboração em participar da avaliação de atividade simpática (vários pacientes consentiram em realizar o exame de RM, mas não a prova de atividade simpática), outros fatores interferiram na avaliação simpática, como, por exemplo, local de residência e possibilidade de locomoção em condições adequadas ao exame, desenvolvimento de 
lombocialtalgia em território radicular dos nervos fibulares (o que impossibilita a realização da microneurografia), alergia ao cobre (que impossibilita o contato com a placa de cobre do pré-amplificador), e intolerância à retirada de medicação antihipertensiva (seja por aumento de pressão arterial e/ou sintomatologia subjetiva).

A sequiência do protocolo está representada graficamente na Figura 2 (pág 18).

\subsection{Avaliação Clínica}

O diagnóstico de HAS foi feito por medidas repetidas de pressão arterial (PA), utilizando de método auscultatório, mais altas que 140 e $90 \mathrm{mmHg}$ (para sistólica e diastólica), ou valores de PA menores que 140 e 90 durante uso efetivo de medicação antihipertensiva combinados com histórico médico de adequação do diagnóstico de HAS (CHOBANIAN ET AL., 2003).

As medidas de pressão arterial ambulatoriais foram obtidas por método auscultatório, após pelo menos 5 minutos em posição sentado, com o braço nivelado à altura do coração. Foram utilizados esfigmo-manômetros de mercúrio regularmente calibrados e validados, e manguitos adequados às dimensões do braço. Foram considerados valores de pressão sistólica os pontos em que ao menos dois ou mais sons se fizeram audíveis, e valores de pressão sistólica aqueles imediatamente anteriores ao desaparecimento dos sons. Ao menos duas tomadas de valores foram realizadas em cada ocasião.

Os indivíduos foram submetidos a exame físico geral e neurológico completo buscando detecção de condições excludentes do estudo, e realizaram exames complementares: ECG, ecocardiografia transtorácica, radiografia de tórax e 
laboratoriais de sangue (hemograma, dosagem sérica de creatinina, ácido úrico, sódio, potássio, colesterol, triglicérides e glicose).

Foram excluídos do protocolo indivíduos com doença arterial sistêmica e outras doenças ou condições sistêmicas ou neurológica prévias ou presentes capazes de alterar os resultados de IRM e ASNM, tais como antecedente pessoal, sintomas ou sinais de: doença arterial periférica, coronariopatia, doença vascular cerebral isquêmica ou hemorrágica, insuficiência cardíaca, diabetes mellitus, neuropatia periférica ou radiculopatia, claustrofobia, e outras condições incidentais.

\subsection{Exame de Ressonância Magnética e divisão dos grupos}

Os estudos foram realizados no Instituto de Radiologia do HCFMUSP, em aparelho de 1,5 Tesla (GE - Horizon). Foi utilizada bobina de crânio, sendo realizados em cada indivíduo pelo menos 3 seqüências em "Fast Spin-echo": sagital T1 localizador, axial T2 e coronal T2 com cortes de 3mm de espessura e intervalos entre os cortes de $1 \mathrm{~mm}$. O campo de visão foi de 20, a matriz variou de 196256x256, e o número de excitações foi de 2-4. A qualidade do sinal obtido foi considerada adequada em todos os exames.

As imagens foram gravadas em disco óptico, e a análise feita por 2 radiologistas. Foi considerada como sinal de $\mathrm{CNV}$ a presença de imagem vascular adjacente à área RVL do bulbo; quando esta imagem era acompanhada de deformação anatômica do bulbo como aparente decorrência desta imagem, o achado

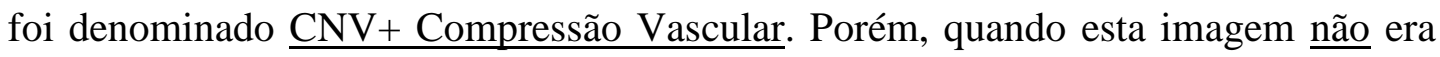
acompanhada de deformação anatômica do bulbo o achado foi denominado $\underline{\mathrm{CNV}+}$ 
Contato Vascular. A presença de deformação anatômica sem imagem vascular não foi considerada como sinal de CNV.

\subsection{Avaliação de Atividade Simpática}

As avaliações de atividade simpática foram realizadas no Laboratório da Unidade de Hipertensão do InCor, após retirada de medicação anti-hipertensiva de acordo com o tempo de ação de cada droga, para evitar interferência na coleta de dados. As retiradas de medicações foram feitas de forma escalonada e gradual para evitar rebote pressórico, e monitoradas com medidas repetidas de PA e inquirição direta via retorno ambulatorial ou contato telefônico sobre queixas ou sintomas adversos. As medicações antihipertensivas eram reintroduzidas caso houvesse sintomatologia atribuível ou agravável pelo aumento de PA, ou se os níveis pressóricos entrassem em faixa potencialmente danosa de forma aguda ao indivíduo.

\subsubsection{Registro dos sinais}

O protocolo de avaliação da atividade simpática constou da monitorização da pressão arterial, freqüência cardíaca, e ASNM, tanto em níveis basais como em resposta ao estímulo hipotérmico ("Teste do Gelo") e ao exercício isométrico ("Hand Grip").

A pressão arterial foi monitorizada de forma não invasiva pelo método oscilométrico (Finapress - Ohmeda 2300, Ohmeda, Englewood, Colorado), e concomitantemente foi feita monitorização da freqüência cardíaca utilizando eletrocardiograma em derivação DII. Os dados foram registrados em microcomputador acoplado a um conversor de sinais (Stemtech, Inc.). O registro e 
análise de sinal (curva de pressão e eletrocardiograma) foram feitas através do programa AT/MCA-CODAS (DATAQ Instruments, Inc., Akron, Ohio 44333, USA), com uma freqüência de amostragem de 500 a $750 \mathrm{~Hz}$ por canal.

A partir do registro das curvas de pressão arterial batimento a batimento foram analisadas as médias e desvio padrão, para cada período estudado, das pressões arteriais sistólica e diástólica e da freqüência cardíaca. Os dados referentes à pressão arterial e freqüência cardíaca foram gerados e analisados em planilha do programa Excel (Microsoft).

A atividade nervosa simpática muscular foi avaliada pela técnica de registro direto de multiunidade da via pós-gangliônica eferente, no fascículo nervoso muscular do nervo fibular, imediatamente inferior à cabeça da fíbula (técnica já validada e empregada regularmente no Laboratório da Unidade de Hipertensão) (DELIUS et al., 1972; VALLBO, 1979; MANCIA et al., 1998; GRASSI et al., 1999). Os registros foram obtidos pela implantação de um micro eletrodo no nervo fibular e de um eletrodo referência a aproximadamente $1 \mathrm{~cm}$ de distância do primeiro. Os eletrodos foram conectados a um pré-amplificador e o sinal do nervo foi alimentado utilizando um filtro passa-banda, e em seguida foi dirigido a um discriminador de amplitude para armazenagem em osciloscópio e em caixa de som. Para fins de registro e análise, o neurograma filtrado foi alimentado através de um integrador de capacitância-resistência para a obtenção da voltagem média da atividade neural.

O sinal elétrico obtido foi registrado em papel milimetrado, e foi feita contagem de espículas representativas de atividade simpática durante os períodos delimitados para análise, seguindo técnica descrita na literatura e utilizada 
regularmente no Laboratório da Unidade de Hipertensão do Instituto do Coração (DELIUS et al., 1972). Os resultados foram expressos em espículas por minuto (espículas/min).

\subsubsection{Seqüência experimental}

As seqüências experimentais foram iniciadas com a instalação do Finapres e eletrocardiógrafo, sendo conferida a equivalência das medidas obtidas pelo Finapress e pelo método auscultatório. Seguiu-se então a punção do nervo fibular, e, após um período de repouso de 15 minutos para retorno às condições basais de pressão arterial e frequiência cardíaca, foram iniciados os registros basais conjuntos de pressão arterial, freqüência cardíaca e atividade elétrica neural por um período de 10 minutos.

Após o registro basal foram feitos os estímulos, que constaram de:

A - Estímulo Térmico Frio (teste do gelo): logo após o fim do período basal. Os indivíduos fizeram imersão completa da mão até o punho em água gelada (aproximadamente $4^{\circ}$ Celsius) durante 120 segundos ou o máximo de tempo tolerado quando inferior a 120 segundos. Houve repouso de 10 minutos para retorno às condições basais.

B - Exercício Isométrico (utilizando dinamômetro de preensão manual): logo após o repouso de 10 minutos subseqüente ao teste do gelo. Os indivíduos fizeram exercício isométrico dos dedos da mão apertando o dinamômetro e mantendo $30 \%$ da carga voluntária máxima durante 2 minutos (ou pelo máximo de tempo possível se este inferior a 2 minutos). 
Ao final dos estímulos o protocolo de avaliação de atividade simpática foi considerado terminado, e os indivíduos reiniciaram o uso de drogas antihipertensivas. 
Figura 2 - Organograma de seleção de pacientes e realização do protocolo.

\section{Triagem para Inclusão no Estudo}

Pesquisa de fatores de exclusão e aceitação

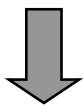

Realização de Ressonância Magnética

Retirada de Medicação Antihipertensiva

Acompanhamento ambulatorial de sinais, sintomas e medidas de PA que indicassem possibilidade de descontrole pressórico

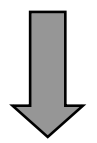

Retorno para realização da Microneurografia Sequência do protocolo:

1.Chegada ao laboratório

2.Instalação de monitores

3.Impactação do microeletrodo

4.Período de repouso - 10 minutos

5.Registro basal - 10 minutos

6. Teste do gelo - 2 minutos

7.Período de repouso - 10 minutos

8.Exercício Isométrico - 2 minutos

9.Fim do protocolo. 


\subsubsection{Análise dos dados}

A) Período basal

Os valores de pressão arterial e frequiência cardíaca foram calculados para o período basal utilizando os valores obtidos batimento a batimento durante o máximo de 10 minutos que antecederam o estímulo do gelo. A contagem de espículas foi realizada em cada minuto durante o período máximo de 10 minutos antecedendo o estímulo do gelo, sendo expressa em espículas por minuto. Foi calculado a média e o erro padrão (EP) dos valores obtidos, que foi considerada representativa do período basal.

B) Estímulos hipotérmico e exercício isométrico

Os períodos de resposta a estímulo foram divididos em períodos consecutivos de 15 segundos a partir do início do estímulo, e foram calculados para cada um destes períodos os valores médios e EP de freqüência cardíaca e PA sistólica e diastólica. Os valores considerados representativos da resposta ao estímulo foram os maiores obtidos após o início do estímulo nesses períodos de 15 segundos, independentemente do tempo decorrido desde o início do estímulo.

A contagem de espículas foi realizada em todo o período de estímulo, sendo expressa em espículas por minuto. Foi considerada para cálculo da resposta a medida mais elevada obtida no primeiro minuto ou segundo minuto de resposta.

A duração média dos estímulos não diferiu entre os grupos. Foi, respectivamente para os grupos CNV-, CNV+ Contato e CNV+Compressão, de $95 \pm$ $12,70 \pm 7$ e $95 \pm 11$ (em segundos \pm erro padrão), durante o gelo, e de $95 \pm 11,100 \pm$ 11 e $107 \pm 8$ (em segundos \pm erro padrão) durante o exercício isométrico. 
A decisão de se considerar amostras de tempo mais longas como representativos da resposta a estímulos para a contagem de espículas foi feita devido ao diferente caráter rítmico dessas variáveis. A atividade simpática normal pode ocorrer tanto em rápidas seqüências de espículas, seguida de períodos de silêncio, como em um ritmo constante, mas levando a uma mesma freqüência por minuto (WALLIN et al., 1993). Por outro lado, freqüência cardíaca e PA fornecem médias confiáveis em intervalos menores, e se alteram de forma representativa também em intervalos menores.

Foram calculados para análise da resposta aos estímulos os valores absolutos, incrementos absolutos e incrementos percentuais das pressões sistólica e diastólica (mmHg), freqüência cardíaca (bpm), e contagem de espícula (espículas/min).

\subsection{Análise Estatística}

Os resultados dos dados clínicos e laboratoriais e da avaliação de atividade simpática foram expressos em média \pm erro padrão da média (EP).

As freqüências de sinais de CNV nos grupos de hipertensos e normotensos foram comparadas pelo teste de qui-quadrado, sendo adotado nível de significância de $5 \%(\alpha=0,05)$, e considerados dois graus de liberdade para comparação dos valores obtidos de qui-quadrado.

Os dados numéricos clínicos (idade, índice de massa corpórea, PA e frequência cardíaca casual, numero de medicações, tempo de hipertensão), de exames complementares e laboratoriais, e os dados de PA, freqüência cardíaca e ASNM obtidos durante o protocolo de avaliação de atividade simpática foram comparados por análise de variância e teste de Tukey modificado. A resposta dos 
grupos aos estímulos durante as avaliações de atividade simpática foi comparada por análise de variância para amostras seqüenciais (KEEPING, 1995). O nível de significância adotado foi de $5 \%(\alpha=0,05)$, e os graus de liberdade do grupo, total e do resíduo foram sempre considerados, nos dados obtidos no protocolo de atividade simpática, de acordo com o número de variáveis disponíveis para cálculo ("N" identificado nas tabelas). 


\section{RESULTADOS}

\subsection{Exames de Ressonância Magnética}

Serão expostos primeiramente os resultados dos 93 exames de imagem por ressonância magnética (IRM) realizados nos 29 indivíduos normotensos e 64 pacientes hipertensos, e no item seguinte os resultados dos exames de IRM realizados naqueles hipertensos que puderam realizar a avaliação de atividade simpática proposta.

Os aspectos característicos dos achados radiológicos de IRM são ilustrados nas fotos $\mathrm{A}, \mathrm{B}$ e C dos anexos, respectivamente, para ausência de compressão neurovascular (CNV), CNV+ Contato Vascular, e CNV+ Compressão Vascular.

\section{A) Resultados da totalidade dos indivíduos e pacientes que realizaram ressonância magnética}

Achados sugestivos de CNV foram encontrados em 9 dos 29 indivíduos normotensos (27,6\%), e em 42 dos 64 indivíduos hipertensos (66,7\%). A distribuição dos achados sugestivos de CNV em normotensos e hipertensos está representada na Tabela 1, e a diferença entre os grupos foi estatisticamente significante (dois graus de liberdade e $\mathrm{p}<0,05)$. 
TABELA 1 - Distribuição dos tipos de achados sugestivos de CNV em normotensos e hipertensos.

\begin{tabular}{cccc}
\hline Grupo & $\begin{array}{c}\text { CNV- } \\
\text { (ausência de CNV) }\end{array}$ & $\begin{array}{c}\text { CNV+ } \\
\text { Contato } \\
\text { Vascular }\end{array}$ & $\begin{array}{c}\text { CNV+ } \\
\text { Compressão Vascular }\end{array}$ \\
\hline Normotensos & $73 \%(21 / 29)$ & $24 \%(7 / 29)$ & $3 \%(1 / 29)$ \\
Hipertensos & $34 \%(22 / 64)$ & $39 \%(25 / 64)$ & $27 \%(17 / 64)$ \\
\hline
\end{tabular}

CNV - compressão neurovascular

Dos 42 pacientes hipertensos, 20 (48\%) apresentaram sinais de CNV no lado esquerdo, 14 (33\%) do lado direito do bulbo RVL, e 8 (19\%) bilateralmente.

Houve um total de 55 achados sugestivos de CNV em 42 pacientes hipertensos, visto que alguns pacientes apresentavam achados bilateralmente ou dois vasos distintos do mesmo lado do bulbo RVL. Os vasos envolvidos nos achados de CNV estão representados na Tabela 2, para os pacientes hipertensos, e na Tabela 3, para os voluntários normotensos. 
TABELA 2 - Distribuição dos vasos envolvidos em cada categoria de achados sugestivos de CNV em pacientes hipertensos.

\begin{tabular}{|c|c|c|}
\hline $\begin{array}{l}\text { Tipo de Achado de IRM } \\
\qquad(\mathrm{n}=55)\end{array}$ & Vaso & $\begin{array}{l}\text { Número de ocorrências } \\
\qquad \mathrm{N}(\%)\end{array}$ \\
\hline \multirow{5}{*}{$\begin{array}{c}\mathrm{CNV}+\text { Contato } \\
\text { Vascular } \\
(\mathrm{n}=34)\end{array}$} & AV Direita & $12(22)$ \\
\hline & AV Esquerda & 7 (13) \\
\hline & ACPI Direita & $3(5)$ \\
\hline & ACPI Esquerda & $11(20)$ \\
\hline & ACAI Esquerda & $1(2)$ \\
\hline \multirow{4}{*}{$\begin{array}{c}\text { CNV+ Compressão } \\
\text { Vascular } \\
(\mathrm{n}=21)\end{array}$} & AV Direita & $8(15)$ \\
\hline & AV Esquerda & $9(16)$ \\
\hline & $\mathrm{AB} *$ & $1(2)$ \\
\hline & Esquerda ACPI & $3(5)$ \\
\hline
\end{tabular}

$\mathrm{AV}$ - artéria vertebral; $\mathrm{AB}$ - artéria basilar; ACAI - artéria cerebelar anterior inferior; ACPI - artéria cerebelar posterior inferior 
TABELA 3 - Distribuição dos vasos envolvidos em cada categoria de achados de CNV em voluntários normotensos.

\begin{tabular}{|c|c|c|}
\hline $\begin{array}{l}\text { Tipo de Achado de IRM } \\
\qquad(\mathrm{n}=9)\end{array}$ & Artéria & $\begin{array}{c}\text { Número de } \\
\text { ocorrências } \\
\mathrm{N}(\%) \\
\end{array}$ \\
\hline \multirow{3}{*}{$\begin{array}{c}\mathrm{CNV}+\text { Contato } \\
\text { Vascular } \\
(\mathrm{n}=8)\end{array}$} & AV Direita & $3(33)$ \\
\hline & AV Esquerda & $3(33)$ \\
\hline & ACPI Esquerda & $2(22)$ \\
\hline $\begin{array}{c}\text { CNV+ Compressão } \\
\text { Vascular } \\
(\mathrm{n}=1)\end{array}$ & AV Esquerda & $1(12)$ \\
\hline
\end{tabular}

AV - artéria vertebral; AB - artéria basilar; ACAI - artéria cerebelar anterior inferior; ACPI - artéria cerebelar posterior inferior

\section{B) Resultados dos exames de ressonância magnética dos pacientes}

\section{hipertensos que realizaram avaliação de atividade simpática}

Segundo o aspecto da IRM foram constituídos três grupos: um por 8 indivíduos com sinais de CNV+ Contato Vascular, o segundo por 8 indivíduos com sinais de CNV+ Compressão Vascular, e o terceiro por 9 indivíduos sem sinais de CNV na IRM (grupo CNV-).

Os dados referentes à distribuição dos indivíduos quanto aos achados de IRM considerados sugestivos de $\mathrm{CNV}$, e quanto à sua distribuição no grupo $\mathrm{CNV}+$ com relação a lado do achado e vaso responsável pela CNV, estão apresentados nas tabelas 4 e 5 . 
TABELA 4 - Distribuição da lateralização do achado sugestivo de CNV no grupo $\mathrm{CNV}+(\mathrm{n}=16)$.

\begin{tabular}{cccc}
\hline Achado sugestivo de CNV (N) & Lado direito & Lado esquerdo & Bilateral \\
\hline Contato vascular (8) & 1 & 5 & 2 \\
Compressão vascular (8) & 3 & 2 & 3 \\
\hline
\end{tabular}

TABELA 5 - Distribuição do vaso relacionado à CNV e lado do bulbo RVL comprometido no grupo $\mathrm{CNV}+(\mathrm{n}=16)$.

\begin{tabular}{cccc}
\hline $\begin{array}{c}\text { Tipo de achado de } \\
\text { CNV }(\mathrm{n}=16)\end{array}$ & Vaso & $\mathrm{N}$ & Lado do achado \\
\hline \multirow{3}{*}{$\begin{array}{c}\text { Contato vascular } \\
(\mathrm{n}=8)\end{array}$} & ACIP esq & 3 & Esq \\
& AV dir e esq & 2 & Dir e Esq \\
& AV esq & 1 & Esq \\
& ACIP dir e AV dir & 1 & Dir \\
Compressão vascular & AV dir & 3 & Dir \\
$(\mathrm{n}=8)$ & AV esq & 2 & Dir \\
& AV dir e esq & 2 & Esq \\
& AV + AB & 1 & Dir e Esq \\
& & & Esq e Dir \\
\hline
\end{tabular}

ACIP - Artéria cerebelar inferior posterior; AV - Artéria vertebral; AB - Artéria basilar 


\subsection{Avaliação clínica}

Os resultados serão apresentados separadamente para os indivíduos hipertensos e normotensos que realizaram IMR e para os pacientes hipertensos que realizaram também avaliação de atividade simpática, com ênfase neste grupo.

\section{A) Todos os indivíduos que realizaram ressonância magnética}

As medias de idade, pressão arterial e tempo de diagnóstico de hipertensão estão expostos na Tabela 6 . A diferença de idade e de pressão arterial entre os grupos de normotensos e hipertensos foi estatisticamente significante $(\mathrm{p}<0,05)$.

TABELA 6 - Características clínicas dos grupos de hipertensos e normotensos que realizaram Ressonância Magnética.

\begin{tabular}{ccc}
\hline Variável & $\begin{array}{c}\text { Normotensos } \\
(\mathrm{N}=29)\end{array}$ & $\begin{array}{c}\text { Hipertensos } \\
(\mathrm{N}=64)\end{array}$ \\
\hline Idade (anos $\pm \mathrm{EP})$ & $34 \pm 7$ & $43 \pm 9$ \\
PA sistólica $(\mathrm{mmHg} \pm \mathrm{EP})$ & $120 \pm 14$ & $150 \pm 13$ \\
PA diastólica $(\mathrm{mmHg} \pm \mathrm{EP})$ & $73 \pm 8$ & $99 \pm 15$ \\
Tempo de HAS (anos $\pm \mathrm{EP})$ & - & $7,4 \pm 6,5$ \\
\hline
\end{tabular}

PA - pressão arterial; HAS - hipertensão arterial 
B) Pacientes hipertensos que realizaram ressonância magnética e prova de atividade simpática

Os valores de pressão arterial sistólica e diastólica, freqüência cardíaca, número de medicações anti-hipertensivas utilizadas, e dosagens séricas de glicose, sódio, potássio, ácido úrico, colesterol total e triglicérides estão apresentados na Tabela 7. Eram semelhantes em ambos os grupos.

TABELA 7 - Valores absolutos de média e desvio padrão da pressão arterial sistólica e diastólica (mmHg), freqüência cardíaca (bpm), número de medicações antihipertensivas em uso (n) e das mensurações séricas de glicose (mg/dL), sódio (mEq/L), potássio $(\mathrm{mEq} / \mathrm{L})$, ácido úrico $(\mathrm{mg} / \mathrm{dL})$, creatinina $(\mathrm{mg} / \mathrm{dL})$, colesterol total $(\mathrm{mg} / \mathrm{dL})$ e triglicérides $(\mathrm{mg} / \mathrm{dL})$.

\begin{tabular}{cccc}
\hline Variável & $\begin{array}{c}\text { CNV- } \\
(\mathrm{n}=9)\end{array}$ & $\begin{array}{c}\text { Contato } \\
(\mathrm{n}=8)\end{array}$ & $\begin{array}{c}\text { Compressão } \\
(\mathrm{n}=8)\end{array}$ \\
\hline PA Sistólica $(\mathrm{mm} \mathrm{Hg})$ & $145 \pm 15$ & $149 \pm 21$ & $150 \pm 11$ \\
PA Diastólica (mm Hg) & $98 \pm 17$ & $97 \pm 13$ & $99 \pm 14$ \\
Freqüência cardíaca (bpm) & $74 \pm 3$ & $75 \pm 3$ & $74 \pm 4$ \\
Número de medicações & $2,3 \pm 0,4$ & $1,6 \pm 0,5$ & $2,5 \pm 0,3$ \\
glicose (mg/dL) & $95 \pm 6$ & $95 \pm 17$ & $99 \pm 6$ \\
Sódio (mEq/L) & $140 \pm 2$ & $138 \pm 2$ & $144 \pm 2$ \\
potássio (mEq/L) & $4,4 \pm 0,4$ & $4,1 \pm 0,4$ & $4,0 \pm 0,4$ \\
Ácido úrico (mg/dL) & $5,6 \pm 1,6$ & $6,7 \pm 5,3$ & $5,9 \pm 1,6$ \\
creatinina (mg/dL) & $0,9 \pm 0,1$ & $0,9 \pm 0,2$ & $0,9 \pm 0,1$ \\
Colesterol total (mg/dL) & $186 \pm 28$ & $194 \pm 48$ & $199 \pm 28$ \\
triglicérides (mg/dl) & $88 \pm 35$ & $175 \pm 103$ & $98 \pm 35$ \\
\hline
\end{tabular}

PA - pressão arterial 
Os dados referentes a media e desvio padrão de idade e índice de massa corpórea, e composição étnica e sexual dos grupos, estão apresentados na tabela 8 .

TABELA 8 - Dados da casuística: idade (média \pm EP), sexo, etnia e índice de massa corpórea (média $\pm \mathbf{E P})$.

\begin{tabular}{|c|c|c|c|c|}
\hline Grupo $(\mathrm{N})$ & Idade & Sexo & Etnia & $\operatorname{IMC}\left(\mathrm{kg} / \mathrm{m}^{2}\right)$ \\
\hline CNV- (9) & $42,8 \pm 2,2$ & $\begin{array}{l}3 \text { masculino } \\
6 \text { feminino }\end{array}$ & $\begin{array}{c}6 \text { brancos } \\
1 \text { negro } \\
2 \text { pardos }\end{array}$ & $34,4 \pm 3,7$ \\
\hline Contato (8) & $38,0 \pm 4,1$ & $\begin{array}{l}4 \text { masculino } \\
4 \text { feminino }\end{array}$ & $\begin{array}{c}4 \text { brancos } \\
2 \text { pardos } \\
2 \text { negros }\end{array}$ & $34,4 \pm 4,6$ \\
\hline Compressão (8) & $46,9 \pm 2,1$ & $\begin{array}{l}3 \text { masculino } \\
5 \text { feminino }\end{array}$ & $\begin{array}{l}5 \text { brancos } \\
2 \text { negros } \\
1 \text { asiático }\end{array}$ & $29,7 \pm 3,1$ \\
\hline
\end{tabular}

\subsection{Atividade Simpática}

Foram comparados as avaliações realizadas nos grupos CNV-, CNV+ Contato Vacular e CNV+ Compressão Vascular, nos períodos basais e em resposta aos estímulos do gelo e do exercício isométrico. Os dados estão apresentados segundo gráficos e tabelas, na seqüência de realização do protocolo e com referência ao número de pacientes que completaram o teste.

Alguns indivíduos não conseguiram realizar os estímulos, e em alguns casos os resultados obtidos não foram computados por apresentarem má qualidade. Desta forma, o número de indivíduos cujos dados foram considerados está representado em cada tabela. 


\subsubsection{Período Basal}

\section{A) Pressão arterial e freqüiência cardíaca (Tabela 9)}

Não houve diferença estatisticamente significante entre os três grupos quando comparados os valores de pressão arterial e freqüência cardíaca no período basal.

TABELA 9 - Pressão arterial $(\mathrm{mmHg})$ e frequência cardíaca $(\mathrm{bpm})$ no período basal.

\begin{tabular}{cccc}
\hline Grupo (N) & PA sistólica & PA diastólica & $\begin{array}{c}\text { Freqüência } \\
\text { cardíaca }\end{array}$ \\
\hline CNV- (9) & $171 \pm 5$ & $110 \pm 5$ & $72 \pm 3$ \\
Contato (8) & $174 \pm 6$ & $108 \pm 7$ & $71 \pm 3$ \\
Compressão (8) & $183 \pm 7$ & $115 \pm 8$ & $81 \pm 7$ \\
\hline
\end{tabular}

PA - pressão arterial

\section{B) Atividade simpática (Tabela 10)}

A atividade simpática basal era significativamente maior no grupo $\mathrm{CNV}+$ Compressão quando comparados os três grupos no período basal. $\mathrm{O}$ grupo $\mathrm{CNV}+$ Contato teve atividade simpática semelhante à do grupo CNV-.

TABELA 10 - Atividade simpática (espículas/min) no período basal.

\begin{tabular}{cl}
\hline Grupo $(\mathrm{N})$ & Espículas \\
\hline CNV- (9) & $21,4 \pm 3,2$ \\
Contato (8) & $17,5 \pm 1,1$ \\
Compressão (8) & $30,4 \pm 3,4 *$ \\
\hline${ }^{*} \mathrm{p}<0,05$ &
\end{tabular}




\subsubsection{Resposta ao estímulo do gelo (Tabela 11).}

\section{A) Pressão arterial sistólica}

Não houve diferença estatisticamente significante entre os três grupos quando comparados os valores de pressão arterial sistólica na resposta ao estímulo do gelo.

TABELA 11 - Resposta da pressão arterial sistólica ao gelo.

\begin{tabular}{cccc}
\hline Grupo (N) & $\begin{array}{c}\text { Basal } \\
(\mathrm{mmHg})\end{array}$ & $\begin{array}{c}\text { PA sistólica } \\
\text { Incremento } \\
(\mathrm{mmHg})\end{array}$ & $\begin{array}{c}\text { Percentual } \\
(\%)\end{array}$ \\
\hline CNV- (9) & $173 \pm 5$ & $20 \pm 5$ & $12 \pm 5$ \\
Contato (6) & $175 \pm 7$ & $16 \pm 5$ & $10 \pm 5$ \\
Compressão (6) & $179 \pm 8$ & $31 \pm 7$ & $17 \pm 7$
\end{tabular}

PA - pressão arterial

\section{B) Pressão arterial diastólica (Tabela 12).}

Não houve diferença estatisticamente significante entre os três grupos quando comparados os valores de pressão arterial diastólica na resposta ao estímulo do gelo.

TABELA 12 - Resposta da pressão arterial diastólica ao gelo.

\begin{tabular}{cccc}
\hline Grupo (N) & $\begin{array}{c}\text { Basal } \\
(\mathrm{mmHg})\end{array}$ & $\begin{array}{c}\text { PA diastólica } \\
\text { Incremento } \\
(\mathrm{mmHg})\end{array}$ & $\begin{array}{c}\text { Percentual } \\
(\%)\end{array}$ \\
\hline CNV- (9) & $110 \pm 5$ & $13 \pm 3$ & $12 \pm 3$ \\
Contato (6) & $116 \pm 10$ & $12 \pm 4$ & $11 \pm 3$ \\
Compressão (6) & $111 \pm 7$ & $18 \pm 4$ & $17 \pm 4$ \\
\hline
\end{tabular}

$\mathrm{PA}$ - pressão arterial 


\section{C) Freqüiência cardíaca (Tabela 13).}

Não houve diferença estatisticamente significante entre os três grupos quando comparados os valores de freqüência cardíaca na resposta ao estímulo do gelo.

TABELA 13 - Resposta da freqüência cardíaca ao gelo.

\begin{tabular}{cccc}
\hline \multirow{2}{*}{ Grupo (N) } & \multicolumn{3}{c}{ Freqüência Cardíaca } \\
Basal & $\begin{array}{c}\text { Incremento } \\
(\mathrm{bpm})\end{array}$ & $\begin{array}{c}\text { Percentual } \\
(\%)\end{array}$ \\
\hline CNV- (9) & $72 \pm 3$ & $17 \pm 4$ & $24 \pm 5$ \\
Contato (6) & $69 \pm 3$ & $17 \pm 3$ & $24 \pm 5$ \\
Compressão (6) & $73 \pm 9$ & $14 \pm 3$ & $21 \pm 6$ \\
\hline
\end{tabular}

\section{D) Atividade simpática (Tabela 14)}

Como já mencionado, a atividade simpática era maior no grupo Compressão, quando comparados aos dois outros grupos $(\mathrm{p}<0,05)$. Todavia, os incrementos absolutos e o aumento percentual não apresentaram diferença estatisticamente significante entre os grupos.

TABELA 14 - Resposta da atividade simpática ao gelo.

\begin{tabular}{|c|c|c|c|}
\hline \multirow[b]{2}{*}{ Grupo $(\mathrm{N})$} & \multicolumn{3}{|c|}{ Atividade Simpática } \\
\hline & $\begin{array}{c}\text { Basal } \\
\text { (espículas/min) }\end{array}$ & $\begin{array}{l}\text { Incremento } \\
\text { (espículas/min) }\end{array}$ & $\begin{array}{c}\text { Percentual } \\
(\%)\end{array}$ \\
\hline CNV- (9) & $21,4 \pm 3,2$ & $13,2 \pm 3,1$ & $83 \pm 20$ \\
\hline Contato (6) & $17,9 \pm 1,1$ & $11,8 \pm 2,1$ & $67 \pm 13$ \\
\hline Compressão (6) & $33,3 \pm 3,8 *$ & $12,9 \pm 3,0$ & $40 \pm 10$ \\
\hline
\end{tabular}




\subsubsection{Resposta ao exercício isométrico}

\section{A) Pressão arterial sistólica (Tabela 15)}

Não houve diferença estatisticamente significante entre os três grupos quando comparados os valores de pressão arterial sistólica.

TABELA 15 - Resposta da pressão arterial sistólica ao exercício isométrico.

\begin{tabular}{cccc}
\hline Grupo (N) & $\begin{array}{c}\text { Basal } \\
(\mathrm{mmHg})\end{array}$ & $\begin{array}{c}\text { PA sistólica } \\
\text { Incremento } \\
(\mathrm{mmHg})\end{array}$ & $\begin{array}{c}\text { Percentual } \\
(\%)\end{array}$ \\
\hline CNV- (8) & $179 \pm 5$ & $20 \pm 5$ & $12 \pm 3$ \\
Contato (7) & $172 \pm 7$ & $12 \pm 2$ & $7 \pm 1$ \\
Compressão (6) & $185 \pm 8$ & $16 \pm 3$ & $8 \pm 1$ \\
\hline
\end{tabular}

$\overline{\mathrm{PA}}$ - pressão arterial

\section{B) Pressão arterial diastólica (Tabela 16)}

Não houve diferença estatisticamente significante entre os grupos quando comparados os valores de pressão arterial sistólica.

TABELA 16 - Resposta da pressão arterial diastólica ao exercício isométrico.

\begin{tabular}{cccc}
\hline Grupo (N) & $\begin{array}{c}\text { Basal } \\
(\mathrm{mmHg})\end{array}$ & $\begin{array}{c}\text { PA diastólica } \\
\text { Incremento } \\
(\mathrm{mmHg})\end{array}$ & $\begin{array}{c}\text { Percentual } \\
(\%)\end{array}$ \\
\hline CNV- (8) & $108 \pm 5$ & $12 \pm 4$ & $12 \pm 4$ \\
Contato (7) & $111 \pm 11$ & $9 \pm 1$ & $9 \pm 2$ \\
Compressão (6) & $115 \pm 7$ & $9 \pm 3$ & $7 \pm 2$ \\
\hline
\end{tabular}

PA - pressão arterial 


\section{C) Freqüência cardíaca (Tabela 17)}

Não houve diferença estatisticamente significante entre os grupos quando comparados os valores de freqüência cardíaca.

TABELA 17 - Resposta da freqüiência cardíaca ao exercício isométrico.

\begin{tabular}{cccc}
\hline \multirow{2}{*}{ Grupo (N) } & \multicolumn{3}{c}{ Freqüência Cardíaca } \\
& $\begin{array}{c}\text { Basal } \\
(\mathrm{bpm})\end{array}$ & $\begin{array}{c}\text { Incremento } \\
(\mathrm{bpm})\end{array}$ & $\begin{array}{c}\text { Percentual } \\
(\%)\end{array}$ \\
\hline CNV- (8) & $76 \pm 9$ & $14 \pm 3$ & $17 \pm 3$ \\
Contato (7) & $72 \pm 4$ & $21 \pm 5$ & $31 \pm 8$ \\
Compressão (6) & $84 \pm 8$ & $8 \pm 3$ & $10 \pm 4$ \\
\hline
\end{tabular}

\section{D) Atividade simpática (Tabela 18)}

Como já mencionado, a atividade simpática era maior no grupo Compressão, quando comparados aos dois outros grupos $(\mathrm{p}<0,05)$. Não houve diferença no incremento absoluto entre os três grupos, mas o incremento percentual era menor no grupo Compressão.

TABELA 18 - Resposta da atividade simpática ao exercício isométrico.

\begin{tabular}{|c|c|c|c|}
\hline \multirow[b]{2}{*}{ Grupo (N) } & \multicolumn{2}{|c|}{ Atividade simpática } & \multirow[b]{2}{*}{$\begin{array}{l}\text { Percentual } \\
\quad(\%)\end{array}$} \\
\hline & $\begin{array}{c}\text { Basal } \\
\text { (espículas/min) }\end{array}$ & $\begin{array}{c}\text { Incremento } \\
\text { (espículas/min } \\
\text { ) }\end{array}$ & \\
\hline CNV- (8) & $21,6 \pm 2,2$ & $7,0 \pm 2,0$ & $40 \pm 11$ \\
\hline Contato (7) & $21,5 \pm 2,0$ & $7,4 \pm 2,0$ & $33 \pm 9$ \\
\hline Compressão (6) & $35,8 \pm 4,1 *$ & $4,4 \pm 2,8$ & $11 \pm 7 *$ \\
\hline
\end{tabular}




\subsection{Sumário dos resultados}

Foram realizados exames de IMR investigando sinais de CNV no bulbo RVL em um total de 93 indivíduos, sendo 64 hipertensos e 29 normotensos. Os resultados dos exames de IMR mostraram que:

1. Existem três grandes grupos de achados de IMR: CNV-, nos quais não há sinais de CNV; CNV+ Contato, em que uma imagem vascular está em contato com o bulbo RVL, sem comprimi-lo; e CNV+ Compressão, em que uma imagem vascular causa nítida compressão no bulbo RVL;

2. Os sinais de CNV no bulbo RVL (grupos Contato e Compressão somados) foram evidenciáveis em $66 \%$ dos hipertensos, mas somente em $27 \%$ dos normotensos, diferença que está de acordo com dados de literatura;

3. O achado de CNV+ tipo Compressão evidenciável em $27 \%$ dos hipertensos e em somente $3 \%$ dos normotensos.

Em 25 pacientes hipertensos (provindos do grupo de 64 pacientes que realizaram IMR) foram realizadas avaliações de atividade simpática pela microneurografia de nervo fibular. Havia 9 indivíduos do grupo CNV-, 8 do grupo CNV+ Contato, e 8 do grupo Compressão. A análise das microneurografias permitiu evidenciar que:

1. A atividade simpática no período basal era significativamente mais elevada no grupo Compressão, mas no grupo com apenas contato a atividade era semelhante à do grupo sem sinais de CNV;

2. Embora a atividade simpática em indivíduos do grupo Compressão fosse significativamente mais elevada que a dos outros grupos no período basal, na 
resposta ao gelo e ao exercício isométrico os incrementos de atividade simpática em valores absolutos não foram diferentes. Em valores percentuais a resposta foi menor no teste do exercício isométrico;

3. Os grupos tinham pressão arterial igualmente elevada do período basal e apresentaram respostas semelhantes ao estímulo do gelo e do exercício isométrico. Também não houve diferenças na freqüência cardíaca. 


\section{DISCUSSÃO}

\subsection{Introdução}

Este estudo tem três aspectos fundamentais a serem ressaltados. Inicialmente, trata-se do primeiro a investigar a prevalência de sinais de CNV no bulbo RVL em uma população sul-americana, tanto em indivíduos hipertensos como em normotensos. Foi encontrada diferença significante entre os dois grupos, e esta diferença está de acordo com dados da literatura.

Posteriormente, observou-se ser o heterogêneo grupo dos pacientes que apresentavam CNV na IMR. A variação nos aspectos de imagem não era adequadamente considerada pela literatura, que tratava o grupo dos portadores de CNV na IMR como homogêneo apesar de diferentes aspectos serem considerados como sugestivos de CNV. Os indivíduos com achados de CNV na IRM foram então separados em dois grupos, segundo o aspecto de compressão mais ou menos acentuada sobre o tecido neural (grupos CNV+ Contato e CNV+ Compressão). Verificou-se então que o aspecto CNV+ Compressão era quase exclusivo dos hipertensos (apareceu em 27\% dos hipertensos e em somente 3\% dos normotensos).

Foram realizadas, a seguir, avaliações da atividade simpática nos hipertensos, utilizando a microneurografia de nervo fibular, com o objetivo de comparar os grupos CNV-, CNV+ Contato e CNV+ Compressão. A correlação dos índices de atividade simpática periférica com os diferentes aspectos radiológicos encontrados na IRM mostrou que o grupo CNV+ Compressão apresentava atividade simpática basal mais elevada que os outros grupos, e que o grupo CNV+ Contato apresentava nível de ASNM semelhante ao do grupo CNV-. 


\subsection{Prevalência de CNV em indivíduos com HAS}

A CNV foi primeiramente descrita em associação com a HAS em humanos por Jannetta (JANNETTA et al., 1979; JANNETTA, 1980), em indivíduos submetidos a cirurgias de descompressão neurovascular para tratamento de entidades como neuralgias e disfunções de nervos cranianos. Posteriormente, o mesmo autor descreveu a evolução de indivíduos que, sendo hipertensos e submetidos à descompressão neurovascular por outros motivos, foram tratados no mesmo ato cirúrgico com descompressão neurovascular do bulbo RVL, sendo descrita melhora inicial significativa nos valores de pressão arterial (JANNETTA et al., 1985a).

Após os relatos iniciais de Jannetta outros métodos foram utilizados para verificar a associação entre HAS e CNV. Em angiografias cerebrais Kleineberg observou $81 \%$ de sinais de CNV em hipertensos, e $41.7 \%$ em normotensos (KLEINEBERG et al., 1991). Em necrópsia Naraghi descreveu 24 indivíduos com hipertensão primária, 10 com hipertensão secundária e 21 normotensos, encontrando CNV no bulbo RVL em todos os indivíduos com HAS primária, em dois normotensos, e em nenhum com HAS secundária (NARAGHI et al., 1992).

O método de imagem por ressonância magnética (IRM) para verificação de sinais de CNV associada à hipertensão demonstrou freqüências de 74 a 90\% de sinais de CNV em grupos com hipertensão arterial primária, de 7 a 22.2\% para grupos normotensos, e de 11 a 16.6\% para grupos com hipertensão secundária (NARAGHI et al., 1994; AKIMURA et al., 1995; PIERPAOLO et al., 1996; MORIMOTO et al., 1997). Esses resultados são compatíveis com os números encontrados por Naraghi utilizando dissecção microanatômica (NARAGHI et al., 1994), e mostram que a alteração vascular encontrada provavelmente não é resultado da exposição crônica a 
níveis pressóricos elevados, visto que grupos com hipertensão secundária tiveram frequiência de CNV semelhante à do grupo de normotenso.

Algumas séries utilizando IRM para investigação de CNV não demonstraram diferença entre hipertensos e normotensos (WATTERS et al., 1996; COLON et al., 1998; SAGLITZ et al., 2002). Em alguns desses estudos, no entanto, houve viés tanto na seleção dos indivíduos, sendo feitos análise de bancos de imagens, como na técnica utilizada na aquisição das imagens de ressonância (WATTERS et al., 1996; COLON et al., 1998).

Em nosso estudo utilizou-se uma técnica especialmente planejada para demonstrar com detalhes a interface entre estruturas vasculares, tecido neural e espaços liquóricos na topografia do bulbo RVL, requisitos fundamentais para detecção de CNV. O estudo foi prospectivo, e a seleção de pacientes foi feita excluindo aqueles que tivessem história clínica, sinais ou sintomas de vasculopatia, central ou periférica.

O resultado dos exames de IRM realizados em normotensos e hipertensos neste estudo mostrou que o aspecto de CNV (seja Contato ou Compressão) está associado à HAS também em nossa população, o que está de acordo com os dados da literatura. A prevalência de sinais de CNV no bulbo RVL na IRM observada em grupos de hipertensos e normotensos (66\% e 27\%, respectivamente) diferia estatisticamente, sendo o nosso estudo o primeiro a investigar a prevalência de CNV em população sul-americana. Embora as médias de idade de hipertensos e normotensos tenham sido estatisticamente diferentes para a população final de 29 normotensos, esta diferença possivelmente não é importante para a comparação das prevalências dos aspectos de CNV, pois a prevalência nos normotensos manteve-se a 
mesma que numa fase anterior do estudo, quando a média de idade era mais alta neste grupo (SENDESKI et al., 2002).

A questão de porque alguns estudos não mostraram diferença na prevalência de sinais de CNV no bulbo RVL de hipertensos e normotensos poderia ser explicada, entre outras causas, pela diferença de etnias na população estudada.

\subsection{Diferentes aspectos de CNV - compressão e contato vascular}

$\mathrm{Na}$ descrição feita por Jannetta em hipertensos submetidos à descompressão neurovascular, vaso anômalo encontrado foi, na maioria dos casos a artéria cerebelar posterior inferior (JANNETTA et al., 1985a). Entretanto, os achados não foram classificados conforme a intensidade da compressão.

O estudo de Naraghi, utilizando necropsia e dissecção microanatômica do bulbo, descreveu três padrões de CNV do bulbo RVL: compressão pela artéria cerebelar posterior inferior (mais comum), pela artéria vertebral ou por ambas (NARAGHI et al., 1992). Também neste caso não se fez referência à intensidade da compressão sobre o tecido neural.

Dentre os estudos utilizando IRM, alguns autores tentaram classificar os achados radiológicos sugestivos de CNV conforme o aspecto anatômico, de forma mais ou menos complexa. Entretanto, os autores que propuseram alguma classificação dos achados de CNV (WATTERS et al., 1996; COLON et al., 1998) não encontraram diferença significativa quando compararam a freqüência de sinais de CNV na IRM entre hipertensos e normotensos, o que, provavelmente, desmotivou-os a aprofundarem as pesquisas e compararem grupos com diferentes 
achados radiológicos. Além disso, as classificações propostas eram descritivas em termos anatômicos, sem aparente intenção de se prestar a correlações funcionais.

A variabilidade possível na anatomia da CNV no bulbo RVL, já relatada nos estudos iniciais de Janetta (JANNETTA, 1980) e nos estudos de necropsia de Naraghi (NARAGHI et al., 1992), foi relegada a plano secundário possivelmente pela utilização da IRM para detecção da CNV, num contexto em que o interesse principal era saber se os hipertensos apresentavam ou não maior prevalência da entidade, e não quais as possibilidades neuroanatômicas ou funcionais do achado.

A questão da heterogeneidade dos achados radiológicos de CNV na IRM em nosso estudo foi percebida ainda em fase inicial (SENDESKI et al., 2002). Nesta fase, foram encontrados sinais de $\mathrm{CNV}$ em $25 \%$ dos normotensos (somente o achado de contato neste grupo), em $65 \%$ dos hipertensos leves e moderados, e em $61 \%$ dos hipertensos graves. Todavia, observou-se grande variedade de achados semelhantes aos verificados em outros estudos e considerados sugestivos de CNV. Ao se considerarem todos estes achados, e na busca por uma classificação que tivesse alguma importância funcional (e não apenas descritiva, como as que foram propostas pelos autores já citados), optou-se por considerar como parâmetro mais importante a intensidade da compressão exercida pelo vaso sobre o parênquima. Não seria possível estipular um parâmetro objetivo (linear ou numérico) de intensidade de compressão de uma estrutura das dimensões das artérias na região do bulbo RVL com base em aspectos de imagem de RM; desta forma, optou-se por dividir o grupo com achados sugestivos de CNV em dois subgrupos, conforme houvesse a presença de uma imagem vascular junto ao bulbo RVL e esta imagem estivesse ou não em associação com deformação do parênquima neural. 
Um fato a ser ressaltado, dentre os resultados deste estudo, é que o aspecto de Compressão parece ser típico e praticamente exclusivo de hipertensos, visto que foi encontrado em somente um de 29 normotensos (3\%) mas em 17 (27\%) de 64 hipertensos. Este fato levantou a hipótese de que indivíduos com diferentes aspectos radiológicos sugestivos de CNV pudessem ter comportamento autonômico diferenciado - supondo que uma compressão mais acentuada pudesse ter maior repercussão sobre o parênquima neural. Portanto, ao se efetuar a divisão dos grupos para realização das provas de atividade simpática considerou-se pertinente que os grupos CNV-, Contato e Compressão tivessem seus resultados analisados em separado.

\subsection{Repercussão clínica da CNV - atividade simpática}

O fato de grupos de hipertensos apresentarem, em algumas séries, taxa de prevalência de CNV semelhante a dos grupos normotensos, não exclui a possibilidade de que em alguns indivíduos destas séries o quadro de HAS tenha relação com a CNV detectada. Da mesma forma é também possível que alguns dos indivíduos normotensos, mas com CNV demonstrada, possuam atividade simpática aumentada e possam vir a tornar-se hipertensos. Alem disso, as séries e relatos de caso cirúrgicos já publicados mostram que existem alguns indivíduos que apresentam, após cirurgia na qual se fez descompressão do bulbo RVL, alterações hemodinânicas favoráveis ao controle da HAS (GEIGER et al., 1998; LEVY et al., 1998; FRANK et al., 2001). Dados publicados até o momento utilizando animais experimentais corroboram a possibilidade de existirem indivíduos cuja HAS tem 
uma relação causal ou de agravamento com a CNV (JANNETTA et al., 1985b; MORIMOTO et al., 1997).

A avaliação da atividade simpática em humanos hipertensos e a sua correlação com a presença ou não de sinais de CNV (a IRM parece, na atualidade, ser o método mais adequado) foi iniciada só recentemente. Desde a primeira série publicada investigando sinais de CNV na IRM de hipertensos (NARAGHI et al., 1994), até a primeira série publicada investigando atividade simpática nesses indivíduos, houve um lapso de 5 anos (MAKINO et al., 1999). Existem várias técnicas destinadas a avaliar quantitativamente a atividade simpática em humanos, algumas mais simples tecnicamente, menos invasivas, mas mais sujeitas a interferências. Foram utilizadas a dosagem de noradrenalina sérica, as medidas isoladas de PA e da freqüência cardíaca, e a análise espectral da variabilidade da freqüência cardíaca (MAKINO et al., 1999; MORISE et al., 2000). Somente o trabalho de Shobel (SCHOBEL et al., 2002), utilizou a ASNM. A dosagem de noradrenalina sérica, embora correlacione-se positivamente com situações fisiológicas que alteram a atividade simpática, como exercício físico, sono e manobras posturais, apresenta reprodutibilidade e sensibilidade menores que a ASNM. Isto ocorre, provavelmente, porque a noradrenalina plasmática corresponde a somente 5-10\% do total liberado pelos terminais nervosos; o nível sérico de noradrenalina depende não só da liberação, mas da utilização pelos tecidos, e da recaptação; a amostragem de circulação venosa periférica não pode ser considerada como representativa da concentração liberada nos terminais nervosos (GRASSI, 1998; MANCIA et al., 1998; GRASSI et al., 1999). A análise espectral da frequiência cardíaca, empregada como principal metodologia nos estudo de Morise e Makino 
(MAKINO et al., 1999; MORISE et al., 2000), utiliza técnicas matemáticas sofisticadas para identificar variações cíclicas na freqüência, determinadas por ritmos impostos pelo sistema nervoso autônomo, e, embora a variabilidade de baixa freqüência (aproximadamente $0,1 \mathrm{~Hz}$ ) seja interpretada como indicador de tônus simpático, esta medida não provê uma medida direta da freqüência de descarga da inervação simpática do coração, sofrendo também influência adicional do nervo vago. A variabilidade de freqüência cardíaca apresenta correlação positiva evidente com outros métodos mais diretos de avaliação simpática - como a ASNM e o "spillover" de noradrenalina pelo coração - somente em situações de ativação simpática por alteração postural, que envolvem ativação de baroreceptores arteriais e dos de baixa pressão. Em situações nas quais os baroreceptores não estão envolvidos, essa correlação é menos consistente, ou quase ausente (GRASSI, 1998; MANCIA et al., 1998; GRASSI et al., 1999). A microneurografia é o único método disponível em humanos para registrar diretamente a atividade eferente simpática pós-ganglionar para a territórios vasculares específicos, podendo ser empregada para estudar o território de inervação muscular ou cutânea do nervo escolhido. Embora represente a atividade simpática de território específico e limitado, apresenta correlação positiva com o "spillover" de noradrenalina nos territórios cardíaco, renal e cerebral em indivíduos saudáveis (WALLIN et al., 1993; JENNINGS, 1998; MANCIA et al., 1998; GRASSI et al., 1999). Sua reprodutibilidade é alta para o mesmo indivíduo em situações distintas, e suas principais desvantagens são a complexidade técnica e a invasividade; ter baixa sensibilidade para situações de hipofunção simpática; não permitir comparação adequada de amplitude de espículas, por ser a amplitude dependente da relação do eletrodo com as fibras do nervo; fornecer dados somente 
aplicáveis a uma situação de laboratório, dificultando extrapolação para outras circunstâncias (GRASSI, 1998; JENNINGS, 1998; MANCIA et al., 1998; GRASSI et al., 1999).

Makino foi o primeiro a relatar a investigação da atividade simpática em indivíduos com HAS e CNV. Comparou a atividade simpática entre dois grupos de hipertensos primários, 14 com e 11 sem CNV na RM. Os níveis plasmáticos de noradrenalina eram significativamente mais altos e a sensibilidade baroreflexa pesquisada com infusão de fenilefrina estava levemente diminuída no grupo com CNV, sugerindo atividade simpática aumentada. Não houve diferença significativa entre os grupos nas respostas de pressão arterial e variabilidade de freqüência cardíaca ao stress mental, frio, "hand grip", manobra de Valsalva, infusão de fenilefrina e teste da clonidina, mas os valores para o grupo com $\mathrm{CNV}$ eram globalmente mais elevados. As avaliações autonômicas foram realizadas com os indivíduos internados em hospital, para padronizar as condições de meio ambiente (incluindo dieta, retirada de medicação, horário de sono e vigília), e os grupos apresentaram dados clínicos semelhantes. Os sinais radiológicos considerados sugestivos de CNV parecem corresponder somente ao aspecto $\mathrm{CNV}+\mathrm{Compressão}$ utilizado em nosso estudo, pois descrevem a presença de alça vascular associada à deformação do bulbo RVL como necessária para considerar o aspecto como sugestivo de CNV. Não são relatados detalhes de provável vaso envolvido, somente do lado do achado (oito indivíduos com sinais de CNV à esquerda, e seis indivíduos, à direita). Não foi descrita nenhuma análise de dados clínicos, hemodinâmicos ou de atividade simpática conforme o tipo de achado radiológico sugestivo de CNV (MAKINO et al., 1999). 
Em 1999 Morimoto publicou o caso de um indivíduo hipertenso primário submetido à descompressão neurovascular de nervo facial indicada primariamente para espasmos hemifaciais refratários, sendo feita também descompressão da CNV de bulbo RVL, detectada na IRM pré-operatória, visando o tratamento da hipertensão. Houve, após a cirurgia, indícios de diminuição global da atividade simpática: diminuição da pressão arterial, queda dos níveis plasmático e urinário de noradrenalina, mudança do padrão variabilidade de freqüência cardíaca de baixa para alta freqüência, e queda de atividade nervosa simpática muscular na microneurografia (MORIMOTO et al., 1999).

Morise, em 2000, relatou a comparação da variabilidade de freqüência cardíaca através da densidade de potência espectral de baixa freqüência ajustada à idade ("age adjusted low-frequency power spectral density" - A-PSD) entre 23 indivíduos com HAS primária, 13 indivíduos com HAS secundaria, e 46 normotensos. A A-PSD, utilizada como índice de tônus simpático, estava significativamente mais elevada em hipertensos primários com $\mathrm{CNV}$ do que naqueles sem CNV e em normotensos com e sem CNV. Não constam na publicação dados referentes aos valores de pressão do grupo de hipertensos com e sem $\mathrm{CNV}$, controle dos níveis pressóricos no momento da avaliação (visto que as avaliações foram feitas durante uso de anti-hipertensivos), e dados clínicos (como idade, índice de massa corpórea, tempo de HAS) dos hipertensos com e sem CNV. Portanto, é difícil considerar esses grupos como homogêneos e comparáveis quanto à "age adjusted low-frequency power spectral density". A técnica utilizada para realizar os exames de IRM consistiu de aquisições em T2 para identificar pontos de CNV, e angiografias tridimensionais em IRM para identificar o provável vaso envolvido. Os 
indivíduos foram classificados somente como apresentando ou não CNV na RM, não sendo possível saber se o achado radiológico de CNV corresponderia a contato ou compressão. Foram descritos os prováveis vasos envolvidos, e o lado, mas sem que fosse feita qualquer correlação clínica, hemodinâmica, ou autonômica (MORISE et al., 2000).

Shobel, em 2002, comparou a ASNM de 21 hipertensos com CNV e 12 hipertensos sem CNV, relatando valores basais e em resposta ao estímulo hipotérmico (manobra do gelo) mais elevados no grupo com CNV. A ASNM foi aferida, no entanto, durante uso de medicação anti-hipertensiva, devido à dificuldade prática de se retirar a medicação de forma segura nos indivíduos com níveis pressóricos muito elevados. Foram estudados indivíduos hipertensos em geral, não sendo formados grupos conforme a gravidade da hipertensão, mas o fato das médias da PA sistólica ambulatorial dos grupos serem diferentes $(158 \pm 28 \mathrm{mmHg}$ no grupo com CNV contra $144 \pm 19 \mathrm{mmHg}$ no grupo sem $\mathrm{CNV}$ ) permite supor que havia nesse grupo hipertensos mais graves, ou sem controle adequado, apesar de estarem medicados. Este fato torna a comparação entre os dois grupos temerária, visto que o controle pressórico menos eficiente no grupo com $\mathrm{CNV}$ poderia explicar, por si só, a diferença verificada na ASNM. Esse controle insatisfatório da PA poderia ter sido compensado por uma retirada gradual e escalonada da medicação, como feita na população do presente estudo. Além disso, as médias de idade diferiam entre os grupos (46 \pm 10 anos no grupo com sinais de CNV contra $39 \pm 13$ no grupo sem sinais de $\mathrm{CNV}$ ), e, embora essa diferença não tenha sido estatisticamente significante, poderia ter contribuído para a diferença na ASNM observada entre os grupo. Os grupos com e sem CNV foram divididos, do ponto de vista radiológico, 
segundo a presença de sinal vascular junto ao bulbo RVL do lado esquerdo somente, e esta descrição parece englobar tanto os achados de contato como de compressão, embora tal informação não esteja explícita no texto. São descritos os prováveis vasos envolvidos, mas os dados clínicos, hemodinâmicos e de atividade autonômica são comparados somente entre os grupos com e sem sinais de CNV (SCHOBEL et al., 2002).

Os estudos publicados até o momento na investigação da atividade simpática em hipertensos, com e sem CNV na RM, são importantes por terem aberto um campo de grande interesse. Entretanto, apresentam seus resultados funcionais divididos somente segundo presença ou ausência de CNV na RM, e, embora seja importante saber se os grupos com CNV apresentam globalmente atividade simpática mais alta que os grupos sem CNV, não devem ser esquecidas as possibilidades de compressão mais ou menos acentuada, por um ou mais vasos, uni ou bilateral, com ou sem repercussão funcional sobre o tecido neural. Deve ser também lembrado que existe uma porcentagem de indivíduos normotensos que apresenta sinais de CNV na RM, e, ao menos pelas evidências disponíveis, não apresentam hiperatividade simpática (MORISE et al., 2000).

O presente estudo é a primeira tentativa de identificar, dentro do grupo considerado como "portador de CNV na RM", os hipertensos que realmente possuem repercussão funcional desta $\mathrm{CNV}$. É o primeiro, também, a realizar a avaliação de atividade simpática após a retirada de medicações anti-hipertensivas - providência que retira a influência do uso de diferentes medicações sobre a atividade simpática. As medidas de PA, tanto as realizadas durante uso de medicação como as obtidas nas 
provas de atividade simpática, não diferiam entre os grupos, e isto mostra que os grupos estavam em situação clínica de controle pressórico semelhante.

O grupo CNV+ Compressão, com sinais de CNV sugerindo uma compressão franca, com deformação anatômica evidente, apresentou-se com ASNM significantemente mais alta durante o período basal. Confirmou-se, deste modo, a hipótese de que um aspecto radiológico sugerindo compressão mais acentuada pode ter maior repercussão funcional sobre o tecido neural: a contagem de espículas por minuto no período basal foi $74 \%$ maior no grupo Compressão em relação ao grupo Contato (30,4 e 17,5 - respectivamente), e 41\% maior no grupo Compressão em relação ao grupo CNV- (30,4 e 21,9 - respectivamente).

Deve ser ressaltado, ainda, que os grupos sem sinais de CNV (CNV-) e com sinais de CNV mostrando apenas contato vascular (CNV+ Contato), sem compressão evidente, apresentaram valores de ASNM, PA e freqüência cardíaca praticamente iguais entre si, não sendo possível demonstrar diferença entre ambos.

\subsection{Resposta a estímulos}

O incremento total decorrente das manobras de estímulo (gelo e exercício isométrico) não foi diferente entre os grupos. A freqüência cardíaca dos grupos foi semelhante tanto durante o período basal como em resposta aos estímulos, o que permite supor que o aumento na $\mathrm{ASNM}$ no grupo $\mathrm{CNV}+$ Compressão não foi influenciado por um aumento isolado da freqüência cardíaca.

Deve ser ressaltado que o grupo Compressão respondeu aos estímulos partindo de um patamar basal 74 e $41 \%$ maior em relação aos grupos Contato e CNV-, o que permite supor que o grupo Compressão possuiria menor "reserva" de 
resposta a estímulos em relação aos grupos com atividade basal mais baixa Este fato é demonstrativo de que o grupo Compressão está com sua reatividade a estímulos preservada, embora não seja possível chegar a esta conclusão a partir de seu aumento relativo - visto que este aumento relativo foi menor que o dos outros grupos.

Também deve ser ressaltado que indivíduos com atividade simpática cronicamente elevada podem apresentar reatividade diminuída por mecanismo de saturação periférica de receptores noradrenérgicos (FREY et al., 1989; ROSS, ). Este fato poderia explicar, em parte, porque os três grupos apresentaram magnitudes de respostas iguais a partir de estados basais diferentes, mas a resposta no grupo $\mathrm{CNV}+$ Compressão foi então proporcionalmente menor que a dos outros grupos, como se viu em seu menor aumento percentual na contagem de espículas da ASNM.

\subsection{Limitações do estudo}

A principal limitação deste estudo é inferir a prevalência de CNV em bulbo RVL em hipertensos a partir de uma população pequena para estudos epidemiológicos. Todavia, deve ser lembrado que os custos de se realizar estudos epidemiológicos prospectivos com exames de IRM são elevados, e a maioria dos outros estudos que investigou a associação entre HAS e CNV investigou populações menores.

Também constitui outro ponto de questionamento o fato de as médias de idade dos grupos CNV- e CNV+ Contato serem numericamente menores (ainda que essa diferença não seja estatisticamente significante), visto que a atividade simpática tende a ser mais alta em grupos de idosos. 
Outra limitação diz respeito à variação étnica da população. Se este fator for considerado como importante para explicar diferenças na prevalência de CNV em estudos realizados em países diversos, a heterogeneidade da população brasileira requereria maior discriminação na inclusão de pacientes em estudos semelhantes a serem realizados no futuro. Todavia, esta possibilidade permanece, por enquanto, apenas como uma hipótese.

\subsection{Importância clínica do estudo}

Os resultados deste estudo, ao identificarem para um aspecto radiológico específico de IMR um comportamento fisiológico próprio, podem fornecer a base fisiopatológica para explicar o porque de alguns indivíduos nos estudos de séries cirúrgicas não apresentarem repercussão positiva da cirurgia (um questionamento levantado pelos pelos autores destas séries ao constatarem que alguns de seus pacientes não apresentavam melhora após a cirurgia). Talvez alguns casos de aspecto radiológico considerado como sugestivo de CNV nesses pacientes correspondesse à classificação CNV+ Contato vascular utilizada em nosso estudo, e, nesse caso, não se esperaria realmente que a manipulação da área RVL para retirar um estímulo excitatório fosse benéfica - visto que não estava realmente ocorrendo estimulação da região.

A decorrência talvez mais importante dos nossos resultados seria a possível definição de um subgrupo, dentro da população de pacientes hipertensos com CNV+, cujas características de evolução clínica, resposta a diferentes terapêuticas medicamentosas, e eventual lesão de órgãos alvo pudesse ter características particulares - visto que a atividade simpática é fator reconhecidamente associado a 
essas circunstâncias (MANCIA et al., 1999; ESLER, 2000) - e, portanto, talvez beneficiável por medidas preventivas ou terapêuticas específicas.

Não existem atualmente estudos investigando peculiaridades clínicas de grupos de hipertensos ou normotensos com CNV identificada no bulbo RVL, e a associação de HAS com CNV não é sequer citada nas recomendações do "National High Pressure Education Program" (CHOBANIAN et al., 2003). A maior investigação deste subgrupo, em vários aspectos, pode levar, no futuro, ao reconhecimento de implicações clínicas práticas da associação entre CNV no bulbo RVL e a HAS. 


\section{CONCLUSÕES}

Os resultados do nosso estudo indicam que: 1) uma parcela da população considerada como portadora de hipertensão primária apresenta uma associação epidemiológica com a compressão neurovascular do bulbo RVL demonstrada na IRM; 2) essa associação é mais evidente nos hipertensos com o aspecto de compressão mais acentuada no bulbo (grupo CNV+ Compressão); 3) no grupo CNV+ Compressão há, também, aumento da atividade simpática (microneurografia), o que não ocorre no grupo em que há somente contato sem sinais de compressão. Portanto, a CNV com característica de comprimir a região bulbar RVL provoca hiperatividade simpática, que pode agravar o quadro hipertensivo. 
Anexo A - Imagem de ressonância magnética em T2, em corte axial na altura do bulbo rostral ventro-lateral, mostrando artérias vertebrais na sua posição normal, longe do bulbo.

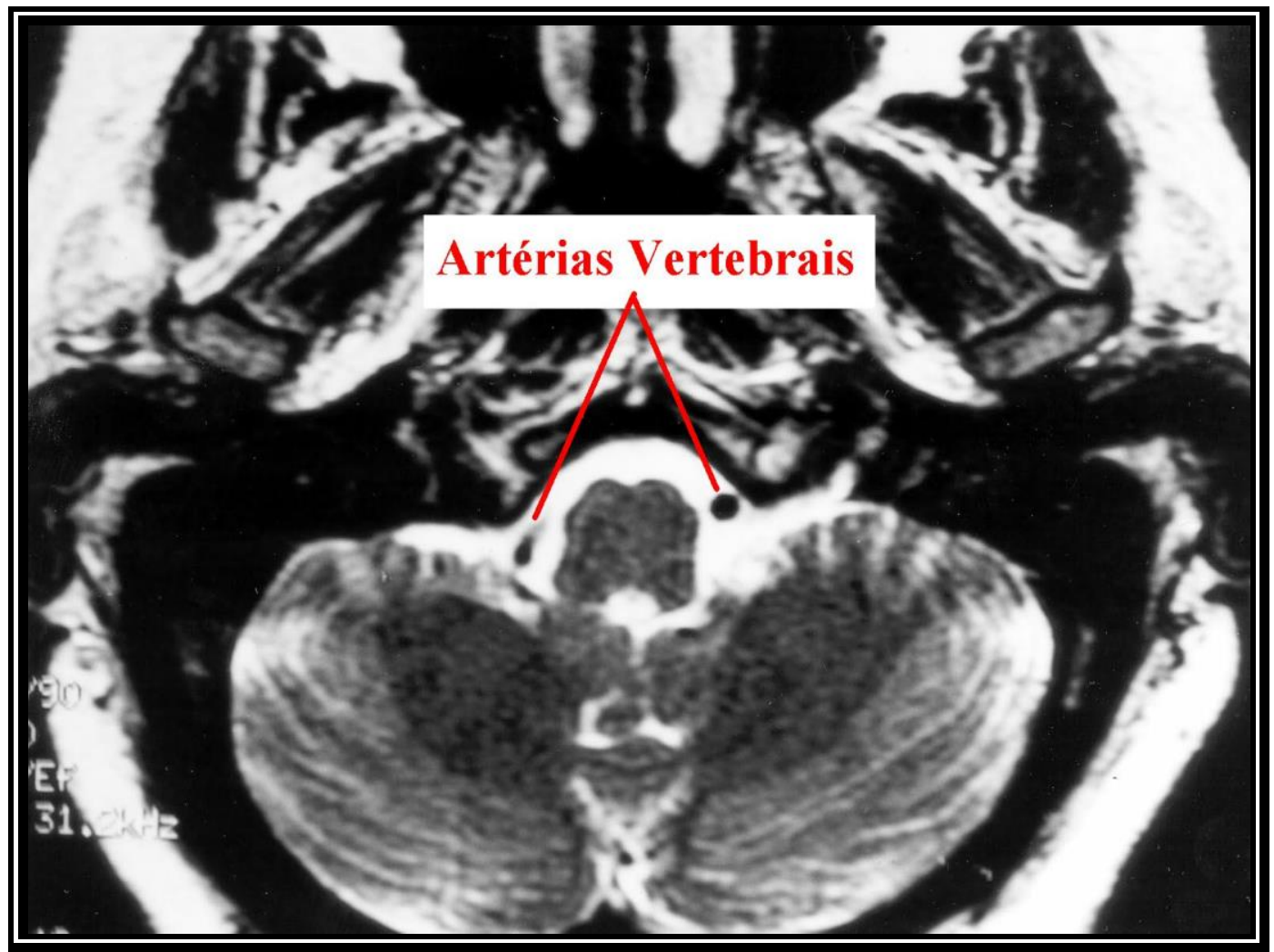


Anexo B - Imagem de Ressonância Magnética em T2, em corte axial na altura do bulbo rostral ventro-lateral, mostrando artéria vertebral esquerda e artéria cerebelar posterior inferior esquerda em contato com o bulbo rostral ventro-lateral, sem causar compressão evidente.

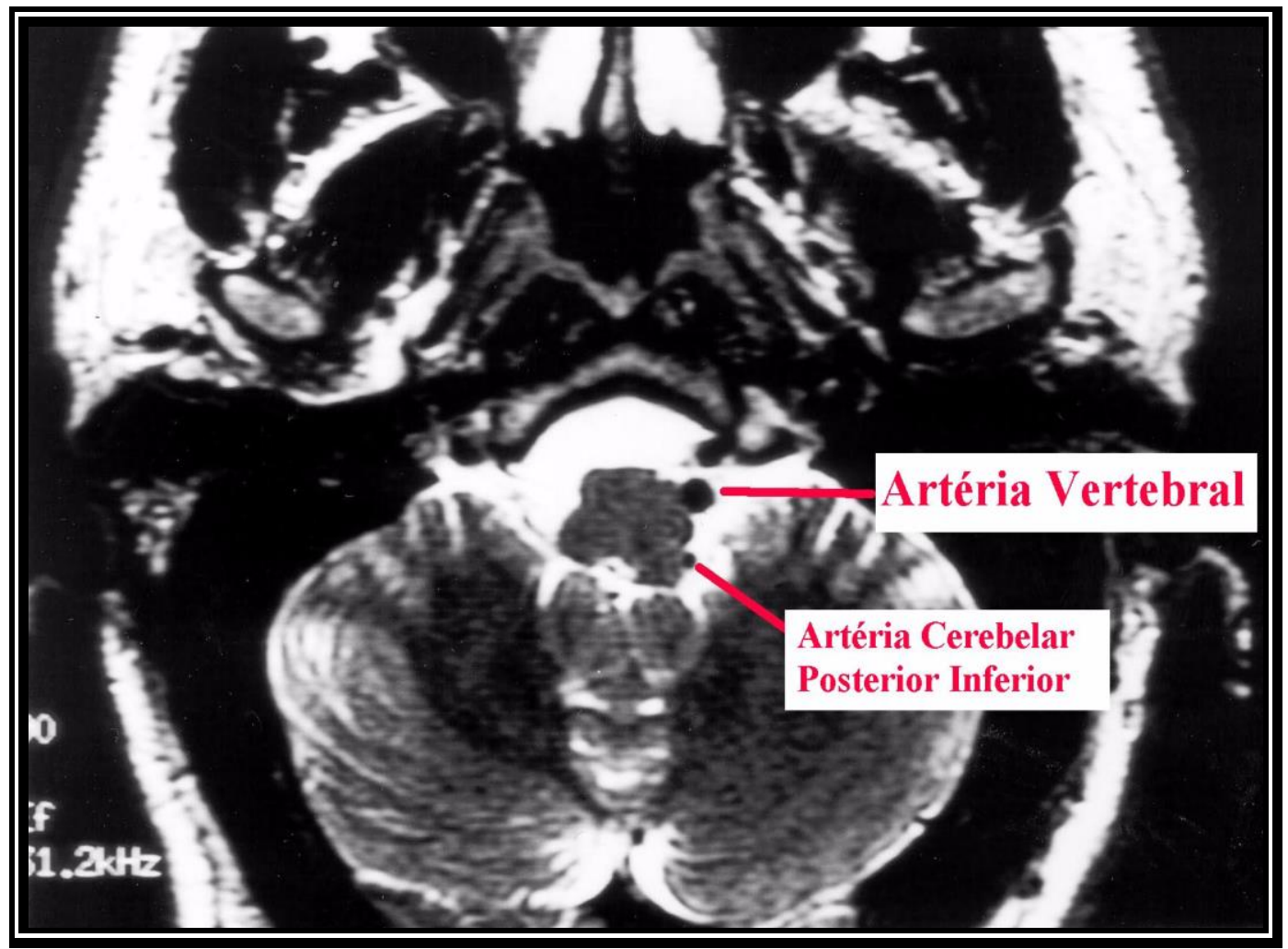


Anexo C - Imagem de Ressonância Magnética em T2, em corte axial na altura do bulbo rostral ventro-lateral, mostrando compressão do bulbo rostral ventro-lateral pela artéria vertebral esquerda.

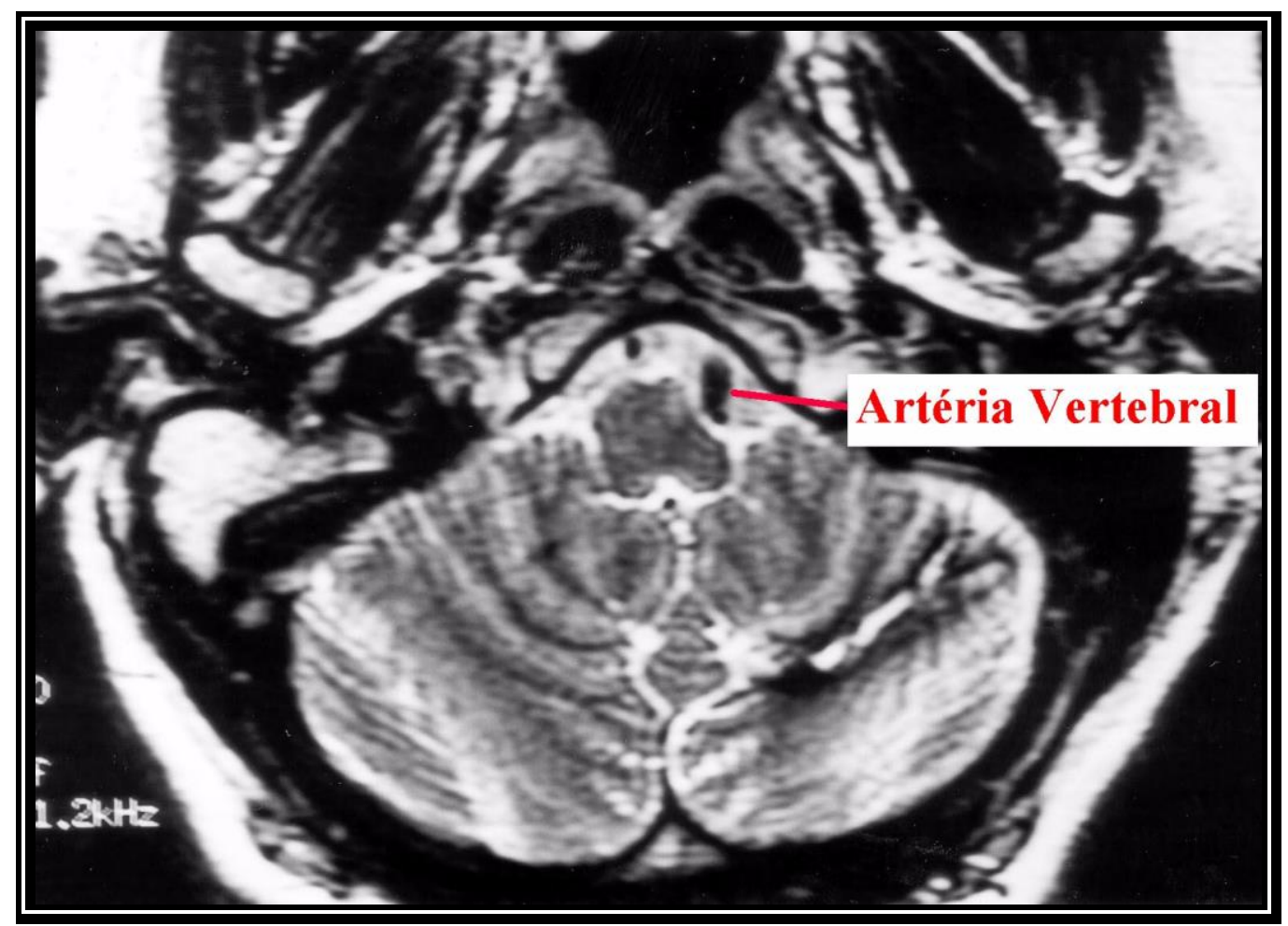


Anexo D - Registro do eletrocardiograma, da curva de pressão arterial e da atividade simpática do nervo fibular em indivíduo do grupo CNV-, no período basal.

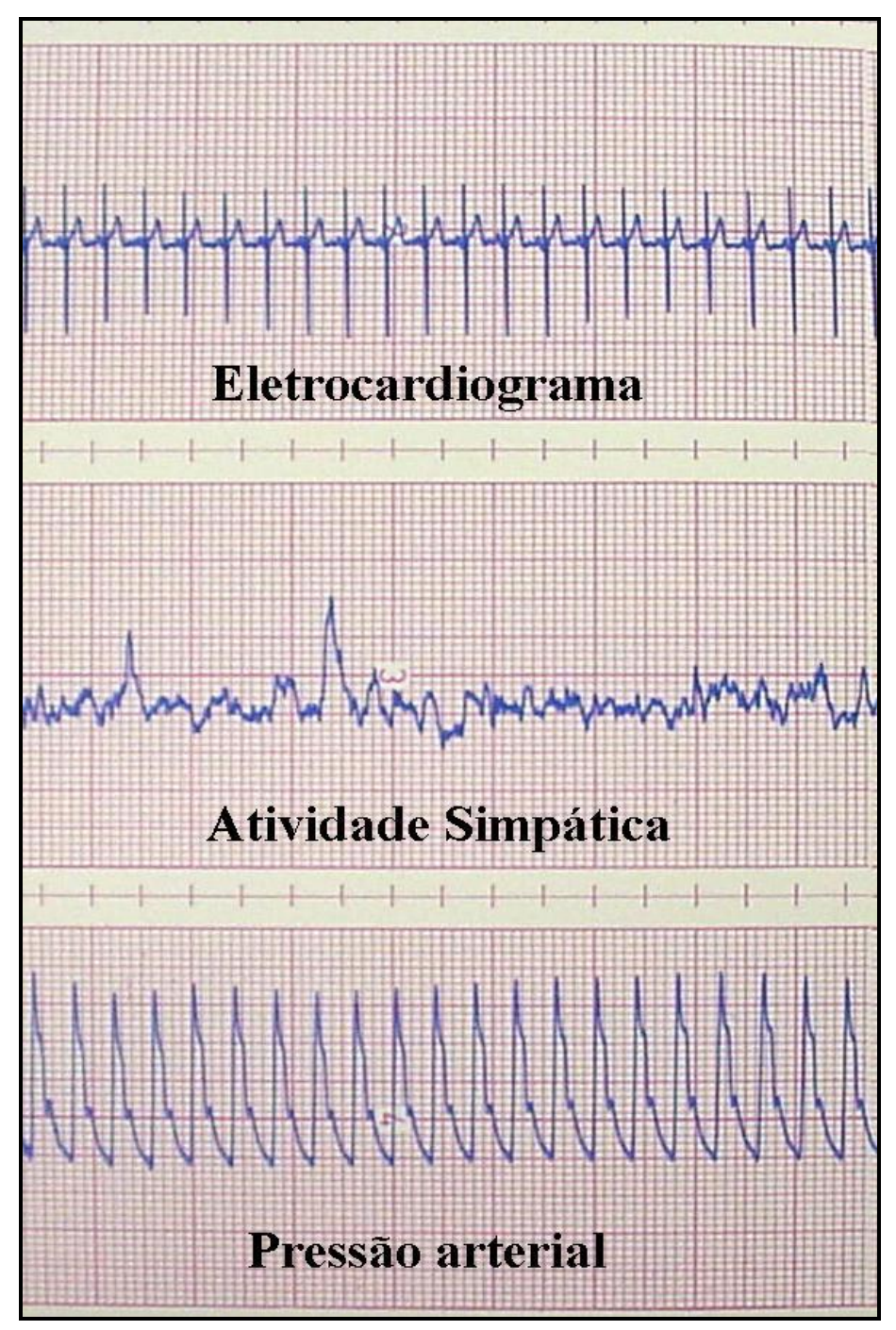


Anexo E - Registro do eletrocardiograma, da curva de pressão arterial e da atividade simpática do nervo fibular em indivíduo do grupo CNV+ Compressão, no período basal.

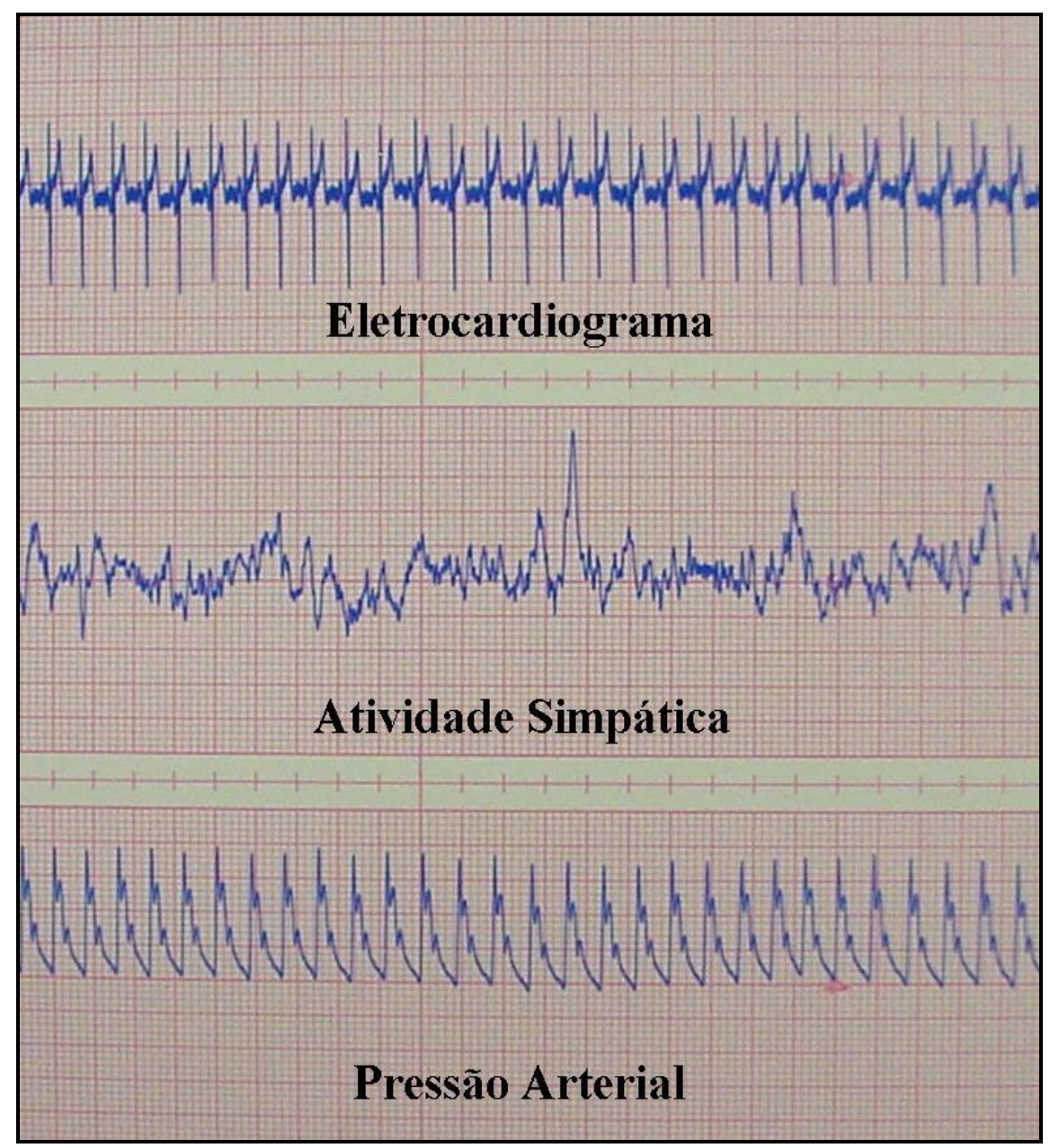


Anexo F - Registro do eletrocardiograma, da curva de pressão arterial e da atividade simpática do nervo fibular em indivíduo do grupo CNV-, na resposta ao estímulo do gelo.

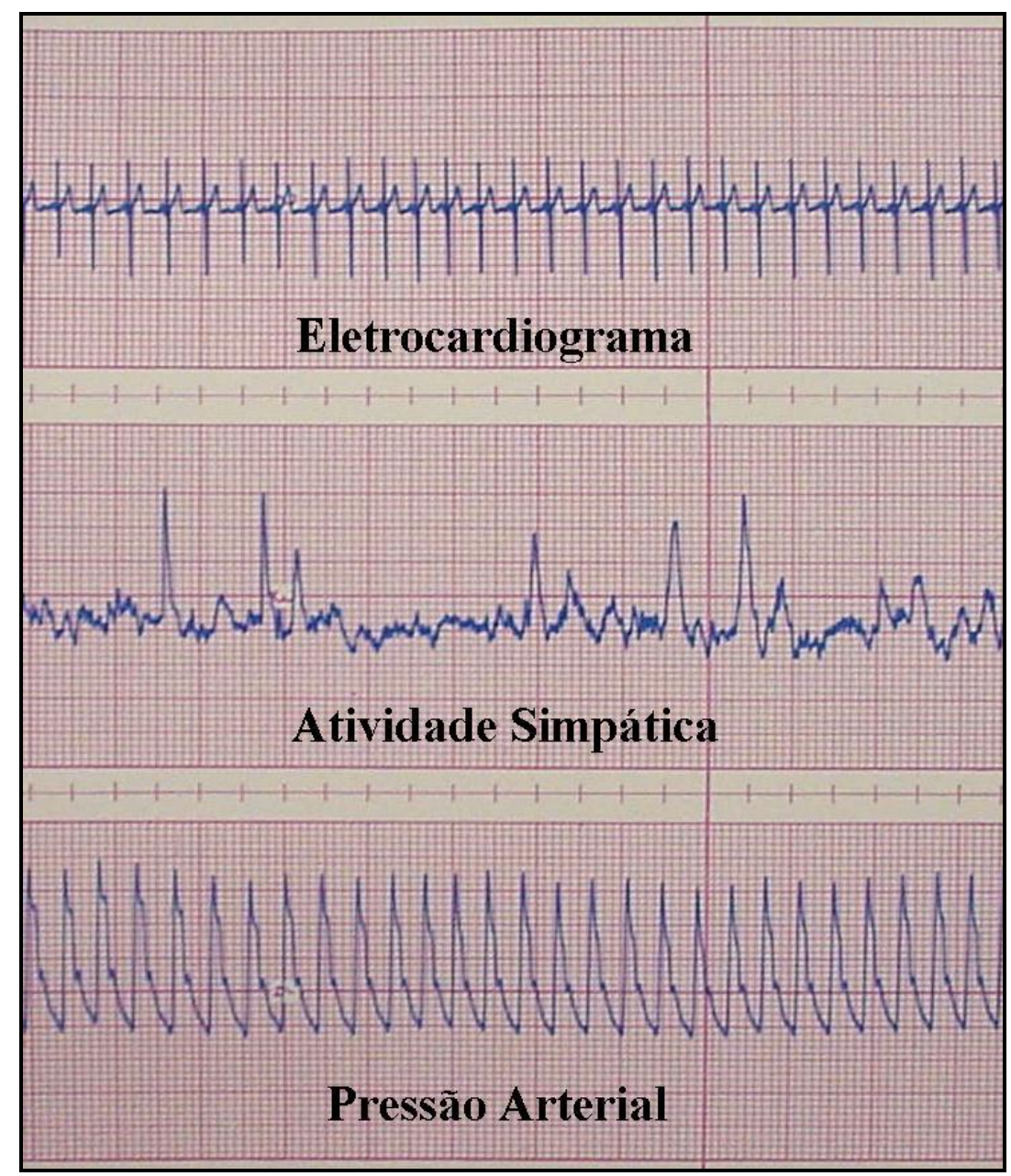


Anexo G - Registro do eletrocardiograma, da curva de pressão arterial e da atividade simpática do nervo fibular em indivíduo do grupo CNV+ Compressão, na resposta ao estímulo do gelo.




Anexo H - Registro do eletrocardiograma, da curva de pressão arterial e da atividade simpática do nervo fibular em indivíduo do grupo CNV-, na resposta ao estímulo do exercício isométrico.

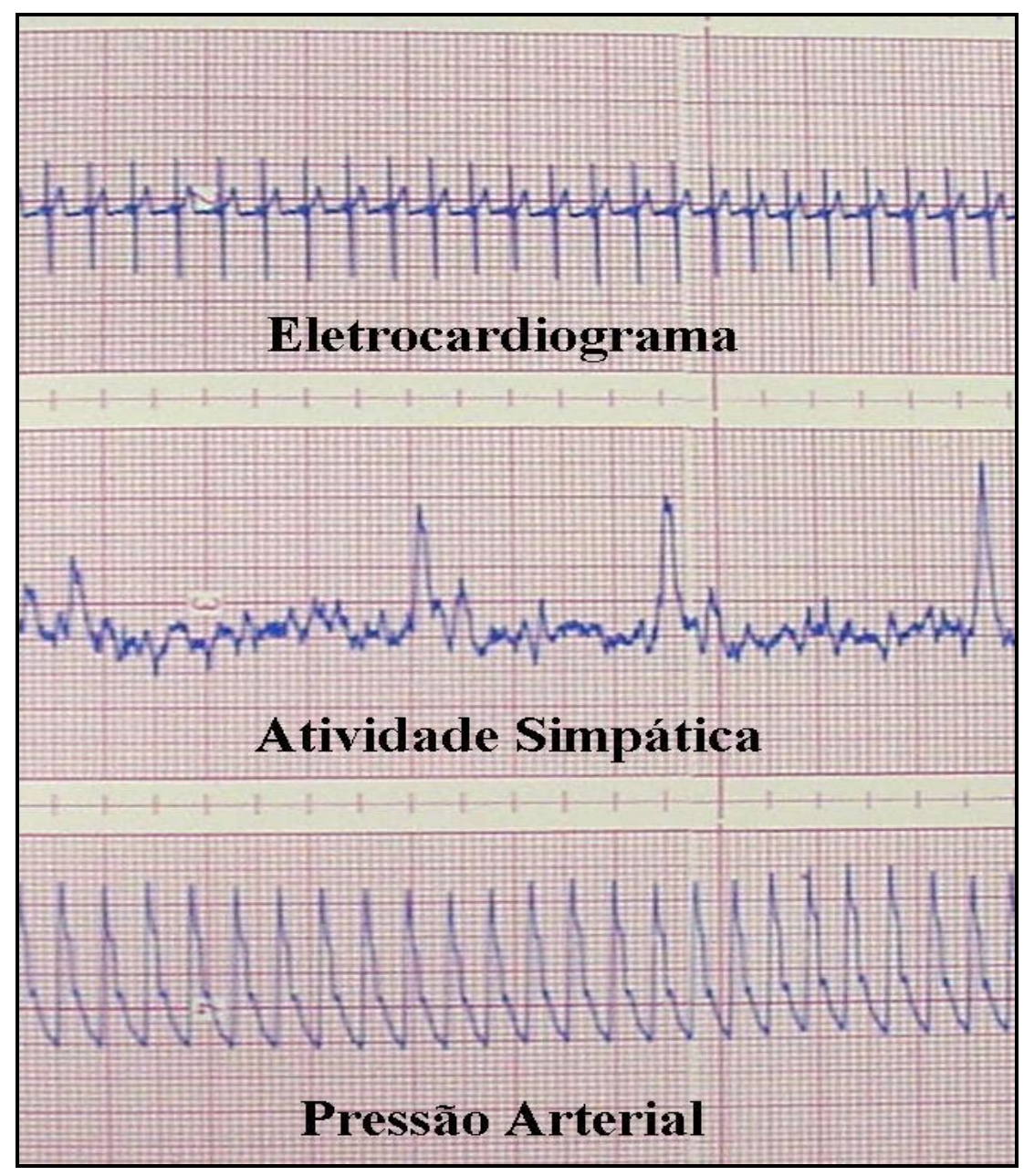


Anexo I - Registro do eletrocardiograma, da curva de pressão arterial e da atividade simpática do nervo fibular em indivíduo do grupo CNV+ Compressão, na resposta ao estímulo do exercício isométrico.

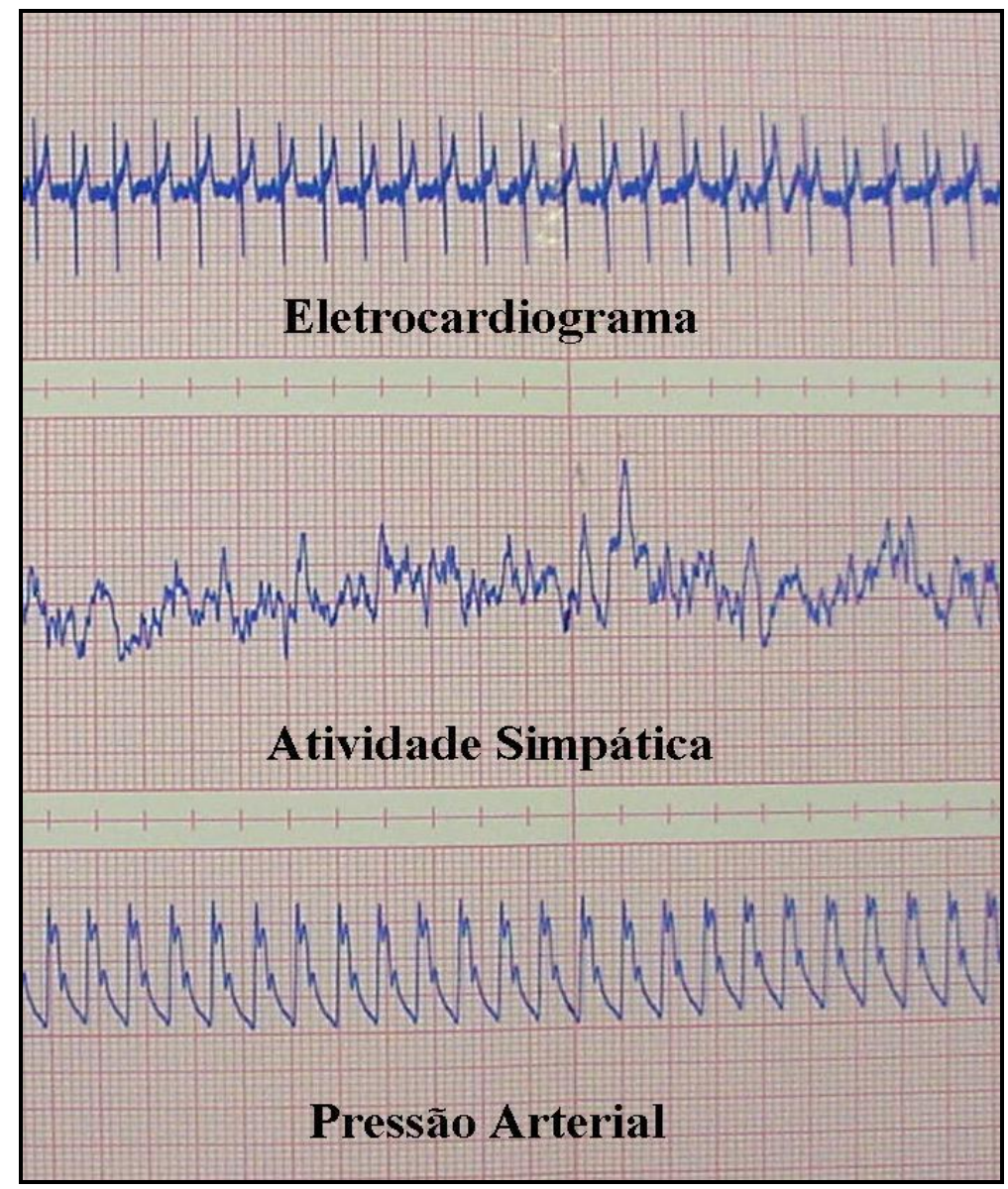


Anexo J - Dados referentes à idade, sexo, raça, índice de massa corpórea, tempo de diagnóstico de HAS, número de drogas anti-hipertensivas, e medidas ambulatoriais de pressão arterial e frequência cardíaca dos indivíduos do grupo CNV-.

\begin{tabular}{cccccccccc}
\hline Nome & $\begin{array}{c}\text { Idade } \\
(\text { anos })\end{array}$ & Sexo & Raça & $\begin{array}{c}\text { IMC } \\
\left(\mathrm{kg} / \mathrm{m}^{2}\right)\end{array}$ & $\begin{array}{c}\text { Tempo de } \\
\text { HAS (anos) }\end{array}$ & DAH & PAS & PAD & FC \\
\hline AFS & 33 & $\mathrm{~F}$ & $\mathrm{Br}$ & 22,0 & 0,5 & 0 & 160 & 100 & 80 \\
$\mathrm{GAC}$ & 45 & $\mathrm{~F}$ & $\mathrm{Br}$ & 32,0 & 10 & 3 & 154 & 114 & 82 \\
$\mathrm{HO}$ & 54 & $\mathrm{~F}$ & $\mathrm{Pd}$ & 30,1 & 10 & 2 & 125 & 80 & 80 \\
$\mathrm{JPR}$ & 41 & $\mathrm{~F}$ & $\mathrm{Ng}$ & 28,2 & 4 & 2 & 140 & 90 & 80 \\
$\mathrm{JLO}$ & 38 & $\mathrm{M}$ & $\mathrm{Pd}$ & 28,6 & 18 & 2 & 135 & 100 & 67 \\
$\mathrm{MO}$ & 42 & $\mathrm{~F}$ & $\mathrm{Br}$ & 35,5 & 13 & 2 & 150 & 106 & 74 \\
NRCS & 49 & $\mathrm{~F}$ & $\mathrm{Br}$ & 27,4 & 14 & 5 & 141 & 86 & 62 \\
OBS & 47 & $\mathrm{M}$ & $\mathrm{Br}$ & 21,8 & 4 & 3 & 171 & 128 & 66 \\
PPSS & 36 & $\mathrm{M}$ & $\mathrm{Br}$ & 22,9 & 3 & 1 & 128 & 75 & 80 \\
\hline
\end{tabular}

IMC - índice de massa corpórea; HAS - hipertensão arterial sistêmica; DAH número de drogas antihipertensivas; PAS - pressão arterial sistólica; PAD - pressão arterial diastólica; FC - frequência cardíaca 
Anexo K - Dados referentes à idade, sexo, raça, índice de massa corpórea, tempo de diagnóstico de HAS, número de drogas anti-hipertensivas, e medidas ambulatoriais de pressão arterial e frequência cardíaca dos indivíduos do grupo CNV+ Contato Vascular.

\begin{tabular}{cccccccccc}
\hline Nome & $\begin{array}{c}\text { Idade } \\
\text { (anos) }\end{array}$ & Sexo & Raça & $\begin{array}{c}\text { IMC } \\
(\mathrm{kg} / \mathrm{m} 2)\end{array}$ & $\begin{array}{c}\text { Tempo } \\
\text { de HAS }\end{array}$ & DAH & PAS & PAD & FC \\
\hline EMM & 45 & $\mathrm{M}$ & $\mathrm{Br}$ & 24,0 & 11,0 & 2 & 140 & 100 & 80 \\
FLS & 33 & $\mathrm{M}$ & $\mathrm{Ng}$ & 25,8 & 0,1 & 0 & 140 & 114 & 66 \\
LAPC & 23 & $\mathrm{M}$ & $\mathrm{Br}$ & 24,2 & 4,0 & 1 & 150 & 95 & 84 \\
MBF & 55 & $\mathrm{~F}$ & $\mathrm{Br}$ & 26,3 & 23,0 & 4 & 141 & 90 & 62 \\
MDS & 48 & $\mathrm{~F}$ & $\mathrm{Br}$ & 22,8 & 0,0 & 2 & 137 & 90 & 70 \\
PSM & 38 & $\mathrm{M}$ & $\mathrm{Br}$ & 23,6 & 4,0 & 1 & 123 & 80 & 85 \\
VMJ & 40 & $\mathrm{~F}$ & $\mathrm{Pd}$ & 26,5 & 18,0 & 3 & 165 & 100 & 80 \\
VOF & 22 & $\mathrm{~F}$ & $\mathrm{Ng}$ & 30,1 & 1,0 & 0 & 141 & 87 & 74 \\
\hline
\end{tabular}

IMC - índice de massa corpórea; HAS - hipertensão arterial sistêmica; DAH número de drogas antihipertensivas; PAS - pressão arterial sistólica; PAD - pressão arterial diastólica; FC - frequência cardíaca 
Anexo L - Dados referentes à idade, sexo, raça, índice de massa corpórea, tempo de diagnóstico de HAS, número de drogas anti-hipertensivas, e medidas ambulatoriais de pressão arterial e frequência cardíaca dos indivíduos do grupo CNV+ Compressão vascular.

\begin{tabular}{cccccccccc}
\hline Nome & $\begin{array}{c}\text { Idade } \\
\text { (anos) }\end{array}$ & Sexo & Raça & $\begin{array}{c}\text { IMC } \\
\left(\mathrm{kg} / \mathrm{m}^{2}\right)\end{array}$ & $\begin{array}{c}\text { Tempo de } \\
\text { HAS }\end{array}$ & DAH & PAS & PAD & FC \\
\hline DAN & 42 & $\mathrm{~F}$ & $\mathrm{Pd}$ & 22,0 & 3 & 2 & 129 & 84 & 75 \\
HS & 42 & $\mathrm{M}$ & $\mathrm{Ng}$ & 22,5 & 4 & 2 & 161 & 98 & 88 \\
JFL & 55 & $\mathrm{M}$ & $\mathrm{Br}$ & 24,7 & 14 & 3 & 153 & 102 & 80 \\
MPS & 54 & $\mathrm{M}$ & $\mathrm{Br}$ & 32,5 & 10 & 3 & 152 & 104 & 52 \\
MCA & 46 & $\mathrm{~F}$ & $\mathrm{Br}$ & 24,8 & 24 & 4 & 205 & 129 & 70 \\
MJS & 38 & $\mathrm{~F}$ & $\mathrm{Br}$ & 26,2 & 3 & 1 & 125 & 75 & 76 \\
NM & 50 & $\mathrm{~F}$ & $\mathrm{Or}$ & 21,5 & 17 & 3 & 143 & 96 & 75 \\
SO & 48 & $\mathrm{M}$ & $\mathrm{Br}$ & 30,8 & 5 & 2 & 135 & 83 & 80 \\
\hline
\end{tabular}

IMC - índice de massa corpórea; HAS - hipertensão arterial sistêmica; DAH número de drogas antihipertensivas; PAS - pressão arterial sistólica; PAD - pressão arterial diastólica; FC - frequência cardíaca 
Anexo $\mathrm{M}$ - Dados referentes à média de pressão arterial $(\mathrm{mmHg})$, frequência cardíaca (bpm) e atividade simpática (ASNM - espículas/min) dos indivíduos do grupo CNV- no período basal.

\begin{tabular}{ccccc}
\hline Nome & PAS & PAD & FC & ASNM \\
\hline AFS & 176 & 116 & 82 & 33 \\
GAC & 148 & 102 & 76 & 15 \\
HO & 169 & 88 & 73 & 11 \\
JPR & 180 & 102 & 68 & 27 \\
JLO & 155 & 117 & 84 & 17 \\
MO & 191 & 120 & 68 & 11 \\
NRCS & 181 & 103 & 57 & 15 \\
OBS & 186 & 140 & 72 & 26 \\
PPSS & 168 & 101 & 66 & 38 \\
\hline
\end{tabular}

PAS - pressão arterial sistólica; PAD - pressão arterial diastólica; FC - frequência cardíaca; ASNM - atividade simpática do nervo 
Anexo $\mathrm{N}$ - Dados referentes à média de pressão arterial $(\mathrm{mmHg})$, frequência cardíaca (bpm) e atividade simpática (ASNM - espículas/min) dos indivíduos do grupo CNV+ Contato vascular no período basal.

\begin{tabular}{ccccc}
\hline Nome & PAS & PAD & FC & ASNM \\
\hline EMM & 194 & 115 & 61 & 15 \\
FLS & 168 & 88 & 78 & 14 \\
LAPC & 144 & 88 & 80 & 16 \\
MBF & 184 & 94 & 69 & 22 \\
MDS & 196 & 151 & 78 & 19 \\
PSM & 168 & 101 & 56 & 22 \\
VMJ & 167 & 118 & 71 & 16 \\
VOF & 174 & 112 & 71 & 15 \\
\hline
\end{tabular}

PAS - pressão arterial sistólica; PAD - pressão arterial diastólica; FC - frequência cardíaca; ASNM - atividade simpática do nervo 
Anexo $\mathrm{O}$ - Dados referentes à média de pressão arterial $(\mathrm{mmHg})$, frequência cardíaca (bpm) e atividade simpática (ASNM - espículas/min) dos indivíduos do grupo CNV+ Compressão vascular no período basal.

\begin{tabular}{ccccc}
\hline Nome & PAS & PAD & FC & ASNM \\
\hline DAN & 152 & 92 & 83 & 25 \\
HS & 182 & 121 & 103 & 40 \\
JFL & 199 & 133 & 59 & 24 \\
MCA & 202 & 138 & 66 & 25 \\
MJS & 177 & 95 & 98 & 30 \\
MPS & 190 & 100 & 107 & 48 \\
NO & 208 & 143 & 78 & 19 \\
SO & 155 & 95 & 67 & 33 \\
\hline
\end{tabular}

PAS - pressão arterial sistólica; PAD - pressão arterial diastólica; FC - frequência cardíaca; ASNM - atividade simpática do nervo 
Anexo $\mathrm{P}$ - Dados referentes à média de pressão arterial $(\mathrm{mmHg})$, frequência cardíaca (bpm), atividade simpática (ASNM - espículas/min) e duração do estímulo (seg) dos indivíduos do grupo CNV- na resposta ao estímulo do gelo.

\begin{tabular}{cccccc}
\hline Nome & PAS & PAD & FC & ASNM & Duração \\
\hline AFS & 193 & 141 & 120 & 40 & 48 \\
GAC & 188 & 110 & 96 & 36 & 94 \\
HO & 193 & 100 & 79 & 29 & 120 \\
JPR & 195 & 109 & 82 & 38 & 120 \\
JLO & 161 & 118 & 102 & 40 & 120 \\
MO & 211 & 142 & 93 & 19 & 99 \\
NRCS & 194 & 113 & 73 & 33 & 60 \\
OBS & 193 & 152 & 89 & 46 & 120 \\
PPSS & 213 & 125 & 67 & 31 & 60 \\
\hline
\end{tabular}

PAS - pressão arterial sistólica; PAD - pressão arterial diastólica; FC - frequência cardíaca; ASNM - atividade simpática do nervo 
Anexo Q - Dados referentes à média de pressão arterial $(\mathrm{mmHg})$, frequência cardíaca (bpm), atividade simpática (ASNM - espículas/min) e duração do estímulo (seg) dos indivíduos do grupo CNV+ Contato vascular na resposta ao estímulo do gelo.

\begin{tabular}{cccccc}
\hline Nome & PAS & PAD & FC & ASNM & Duração \\
\hline EMM & 205 & 132 & 78 & 22 & 80 \\
LAPC & 144 & 92 & 86 & 34 & 87 \\
MBF & 196 & 102 & 92 & 37 & 72 \\
MDS & 209 & 158 & 99 & 22 & 79 \\
PSM & 178 & 105 & 64 & 34 & 85 \\
VOF & 190 & 125 & 86 & 32 & 46 \\
\hline
\end{tabular}

PAS - pressão arterial sistólica; PAD - pressão arterial diastólica; FC - frequência cardíaca; ASNM - atividade simpática do nervo 
Anexo $\mathrm{R}$ - Dados referentes à média de pressão arterial $(\mathrm{mmHg})$, frequência cardíaca (bpm), atividade simpática (ASNM - espículas/min) e duração do estímulo (seg) dos indivíduos do grupo CNV+ Compressão vascular na resposta ao estímulo do gelo.

\begin{tabular}{cccccc}
\hline Nome & PAS & PAD & FC & ASNM & Duração \\
\hline HS & 237 & 157 & 105 & 49 & 120 \\
JFL & 211 & 146 & 72 & 26 & 68 \\
MCA & 222 & 145 & 90 & 36 & 106 \\
MJS & 221 & 118 & 112 & 53 & 52 \\
MPS & 239 & 129 & 109 & 60 & 76 \\
SO & 183 & 104 & 74 & 53 & 118 \\
\hline
\end{tabular}

PAS - pressão arterial sistólica; PAD - pressão arterial diastólica; FC - frequência cardíaca; ASNM - atividade simpática do nervo 
Anexo $\mathrm{S}$ - Dados referentes à média de pressão arterial $(\mathrm{mmHg})$, frequência cardíaca (bpm) e atividade simpática (ASNM - espículas/min) dos indivíduos do grupo CNVno período basal pré estímulo do exercício isométrico.

\begin{tabular}{ccccc}
\hline Nome & PAS & PAD & FC & ASNM \\
\hline AFS & 180 & 125 & 103 & 30 \\
GAC & 163 & 97 & 91 & 16 \\
HO & 184 & 90 & 74 & 11 \\
JLO & 182 & 106 & 81 & 26 \\
JPR & 154 & 123 & 87 & 25 \\
MO & 190 & 121 & 73 & 22 \\
NRCS & 182 & 90 & 82 & 27 \\
PPSS & 202 & 112 & 63 & 19 \\
\hline
\end{tabular}

PAS - pressão arterial sistólica; PAD - pressão arterial diastólica; FC - frequência cardíaca; ASNM - atividade simpática do nervo 
Anexo $\mathrm{T}$ - Dados referentes à média de pressão arterial $(\mathrm{mmHg})$, frequência cardíaca (bpm) e atividade simpática (ASNM - espículas/min) dos indivíduos do grupo CNV+ Contato vascular no período basal pré estímulo do exercício isométrico.

\begin{tabular}{ccccc}
\hline Nome & PAS & PAD & FC & ASNM \\
\hline EMM & 190 & 114 & 72 & 20 \\
LAPC & 144 & 86 & 80 & 23 \\
MBF & 186 & 96 & 85 & 23 \\
MDS & 188 & 152 & 69 & 20 \\
PSM & 161 & 102 & 61 & 27 \\
VMJ & 177 & 135 & 71 & 12 \\
VOF & 179 & 92 & 64 & 27 \\
\hline
\end{tabular}

PAS - pressão arterial sistólica; PAD - pressão arterial diastólica; FC - frequência cardíaca; ASNM - atividade simpática do nervo 
Anexo U - Dados referentes à média de pressão arterial $(\mathrm{mmHg})$, frequência cardíaca (bpm) e atividade simpática (ASNM - espículas/min) dos indivíduos do grupo CNV+ Compressão vascular no período basal pré estímulo do exercício isométrico.

\begin{tabular}{ccccc}
\hline Nome & PAS & PAD & FC & ASNM \\
\hline HS & 194 & 131 & 100 & 38 \\
JFL & 200 & 136 & 68 & 21 \\
MCA & 195 & 134 & 69 & 37 \\
MJS & 188 & 96 & 103 & 36 \\
MPS & 209 & 108 & 101 & 68 \\
SO & 157 & 96 & 63 & 41 \\
\hline
\end{tabular}

PAS - pressão arterial sistólica; PAD - pressão arterial diastólica; FC - frequência cardíaca; ASNM - atividade simpática do nervo 
Anexo V - Dados referentes à média de pressão arterial $(\mathrm{mmHg})$, frequência cardíaca (bpm), atividade simpática (ASNM - espículas/min) e duração do estímulo dos indivíduos do grupo CNV- na resposta ao estímulo do exercício isométrico.

\begin{tabular}{cccccc}
\hline Nome & PAS & PAD & FC & ASNM & Duração \\
\hline AFS & 214 & 153 & 132 & 38 & 87 \\
GAC & 196 & 128 & 106 & 27 & 120 \\
HONORIO & 188 & 94 & 80 & 16 & 57 \\
JLO & 195 & 107 & 102 & 25 & 120 \\
JPR & 159 & 126 & 95 & 37 & 112 \\
MO & 199 & 129 & 84 & 22 & 114 \\
NRCS & 227 & 113 & 96 & 37 & 63 \\
PPSS & 216 & 113 & 73 & 34 & 55 \\
\hline
\end{tabular}

PAS - pressão arterial sistólica; PAD - pressão arterial diastólica; FC - frequência cardíaca; ASNM - atividade simpática do nervo 
Anexo X - Dados referentes à média de pressão arterial $(\mathrm{mmHg})$, frequência cardíaca (bpm), atividade simpática (ASNM - espículas/min) e duração do estímulo (seg) dos indivíduos do grupo CNV+ Contato vascular na resposta ao estímulo do exercício isométrico.

\begin{tabular}{cccccc}
\hline Nome & PAS & PAD & FC & ASNM & Duração \\
\hline EMM & 201 & 130 & 80 & 30 & 60 \\
LAPC & 157 & 98 & 94 & 30 & 120 \\
MDS & 196 & 103 & 101 & 38 & 120 \\
MBF & 201 & 149 & 93 & 24 & 114 \\
PSM & 179 & 112 & 72 & 29 & 105 \\
VMJ & 183 & 120 & 92 & 12 & 60 \\
VOF & 186 & 102 & 106 & 39 & 120 \\
\hline
\end{tabular}

PAS - pressão arterial sistólica; PAD - pressão arterial diastólica; FC - frequência cardíaca; ASNM - atividade simpática do nervo 
Anexo $\mathrm{Y}$ - Dados referentes à média de pressão arterial $(\mathrm{mmHg})$, frequência cardíaca (bpm), atividade simpática (ASNM - espículas/min) e duração do estímulo (seg) dos indivíduos do grupo CNV+Compressão vascular na resposta ao estímulo do exercício isométrico.

\begin{tabular}{cccccc}
\hline Nome & PAS & PAD & FC & ASNM & Duração \\
\hline HS & 217 & 145 & 105 & 38 & 120 \\
JFL & 220 & 159 & 67 & 21 & 120 \\
MCA & 220 & 145 & 87 & 37 & 120 \\
MJS & 202 & 102 & 112 & 36 & 80 \\
MPS & 224 & 114 & 107 & 68 & 70 \\
SO & 163 & 97 & 73 & 41 & 118 \\
\hline
\end{tabular}

PAS - pressão arterial sistólica; PAD - pressão arterial diastólica; FC - frequência cardíaca; ASNM - atividade simpática do nervo 


\section{BIBLIOGRAFIA}

AKIMURA, T.; FURUTANI, Y.; JIMI, Y.; SAITO, K.; KASHIWAGI, S.; KATO,

S.; ITO, H. Essential hypertension and neurovascular compression at the ventrolateral medulla oblongata: MR evaluation. AJNR Am J Neuroradiol. v.16, n.2, p.401-405, 1995.

BELL, G.M. Intracranial disorders and hypertension. In: Laragh, J.H. and Brenner, B.M., eds. Hypertension: Pathophysiology, Diagnosis, and Management. New York, NY: Raven Press. v.1, p.703-709, 1990.

BRODY, M.J.; VARNER, K.J.; VASQUEZ, E.C.; LEWIS, S.J. Central nervous system and the pathogenesis of hypertension. Sites and mechanisms. Hypertension. v.18, n.5 Suppl III, p.7-12, 1991.

CAMPOS, R.R.; MCALLEN, R.M. Cardiac sympathetic premotor neurons. Am J Physiol. v.272, n.2 Pt 2, p.R615-620, 1997.

CHALMERS, J.; PILOWSKY, P. Brainstem and bulbospinal neurotransmitter systems in the control of blood pressure. J Hypertens. v.9, n.8, p.675-694, 1991.

CHOBANIAN, A.V.B., G.L.; BLACK, H.R.; CUSHMAN, W.C.; GREEN, L.A.; IZZO, J.L. JR.; JONES, D.W.; MATERSON, B.J.; OPARIL, S.; WRIGHT, J.T.JR.; ROCCELLA E.J.; JOINT NATIONAL COMMITTEE ON PREVENTION, DETECTION, EVALUATION, AND TREATMENT OF HIGH BLOOD PRESSURE. NATIONAL HEART, LUNG, AND BLOOD INSTITUTE; NATIONAL HIGH BLOOD PRESSURE EDUCATION PROGRAM COORDINATING COMMITTEE. Seventh report of the Joint 
National Committee on prevention, Detection, Evaluation, and Treatment of High Blood Pressure. Hypertension. v.42, n.6, p.1206-1252, 2003.

COLON, G.P.; QUINT, D.J.; DICKINSON, L.D.; BRUNBERG, J.A.; JAMERSON, K.A.; HOFF, J.T.; ROSS, D.A. Magnetic resonance evaluation of ventrolateral medullary compression in essential hypertension. J Neurosurg. v.88, n.2, p.226-231, 1998.

DELIUS, W.; HAGBARTH, K.E.; HONGELL, A.; WALLIN, B.G. General characteristics of sympathetic activity in human muscle nerves. Acta Physiol Scand. v.84, n.1, p.65-81, 1972.

ESLER, M. The Sympathetic System and Hypertension. Am J Hypertens. v.13, p.99S-105S, 2000.

FRANK, H.; HP, S.; K, H.; H, G.; R, F.; R, N. Long-term results after microvascular decompression in essential hypertension. Stroke. v.32, n.12, p.2950-2955, 2001.

FREY, M.J.; MOLINOFF, P.B. Mechanisms of downregulation of beta-adrenergic receptors: perspective on the role of beta-adrenergic receptors in congestive heart failure. J Cardiovasc Pharmacol. v.14, p.S13-S18, 1989.

GEIGER, H.; NARAGHI, R.; SCHOBEL, H.P.; FRANK, H.; STERZEL, R.B.; FAHLBUSCH, R. Decrease of blood pressure by ventrolateral medullary decompression in essential hypertension. Lancet. v.352, n.9126, p.446-449, 1998.

GRASSI, G. Role of the sympathetic nervous system in human hypertension. J Hypertens. v.16, n.12 Pt 2, p.1979-1987, 1998.

GRASSI, G.; ESLER, M. How to assess sympathetic activity in humans. J Hypertens. v.17, n.6, p.719-734, 1999. 
GUERTZENSTEIN, P.G. Vasodepressor and pressor responses to drugs topically applied to the ventral surface of the brain stem. J Physiol. v.224, n.2, p.84P85P, 1972.

GUERTZENSTEIN, P.G. Blood pressure effects obtained by drugs applied to the ventral surface of the brain stem. J Physiol. v.229, n.2, p.395-408, 1973.

HADDAD, N.; SILVA, M.B.D. Mortality Due to Cardiovascular Disease in Women During the Reproductive Age (15 to 49 years), in the State of São Paulo, Brazil, from 1991 to 1995. Arq Bras Cardiol. v.75, n.5, p.375-379, 2000.

HANSSON, L.; KILANDER, L.; ÖHRVALL, M. Epidemiology of Hypertension. In: Oparil, S. and Weber, M.A., eds. Hypertension. Philadelphia, PY: WB Saunders Company. p.4-20, 2000.

HOROWITZ, M.B. Brainstem compression as a cause of neurogenic hypertension.

Curr Hypertens Rep. v.1, n.3, p.264-267, 1999.

JANNETTA, P.J. Neurovascular compression in cranial nerve and systemic disease. Ann Surg. v.192, n.4, p.518-525, 1980.

JANNETTA, P.J.; GENDELL, H.M. Clinical observations on etiology of essential hypertension. Surg Forum. v.30, p.431-432, 1979.

JANNETTA, P.J.; SEGAL, R.; WOLFSON, S.K., JR. Neurogenic hypertension: etiology and surgical treatment. I. Observations in 53 patients. Ann Surg. v.201, n.3, p.391-398, 1985a.

JANNETTA, P.J.; SEGAL, R.; WOLFSON, S.K., JR.; DUJOVNY, M.; SEMBA, A.; COOK, E.E. Neurogenic hypertension: etiology and surgical treatment. II. Observations in an experimental nonhuman primate model. Ann Surg. v.202, n.2, p.253-261, 1985b. 
JENNINGS, G.L. Noradrenaline spillover and microneurography measurements in patients with primary hypertension. J Hypertens Suppl. v.16, n.3, p.S35-38, 1998.

KEEPING, E.S. Introduction to statistical inference. In: eds. New York: Dover Publications, Inc. p.451, 1995.

KIELY, J.M.; GORDON, F.J. Role of rostral ventrolateral medulla in centrally mediated pressor responses. Am J Physiol. v.267, n.4 Pt 2, p.H1549-1556, 1994.

KLEINEBERG, B.; BECKER, H.; GAAB, M.R. Neurovascular compression and essential hypertension. An angiographic study. Neuroradiology. v.33, n.1, p.28, 1991.

KLEINEBERG, B.; BECKER, H.; GAAB, M.R.; NARAGHI, R. Essential hypertension associated with neurovascular compression: angiographic findings. Neurosurgery. v.30, n.6, p.834-841, 1992.

LEVY, E.I.; CLYDE, B.; MCLAUGHLIN, M.R.; JANNETTA, P.J. Microvascular decompression of the left lateral medulla oblongata for severe refractory neurogenic hypertension. Neurosurgery. v.43, n.1, p.1-6; discussion 6-9, 1998.

LOTUFO, P.A. Mortalidade Precoce por Doenças do Coração no Brasil. Comparação com Outros Países. Arq Bras Cardiol. v.70, n.5, p.321-325, 1998. MAKINO, Y.; KAWANO, Y.; OKUDA, N.; HORIO, T.; IWASHIMA, Y.; YAMADA, N.; TAKAMIYA, M.; TAKISHITA, S. Autonomic function in hypertensive patients with neurovascular compression of the ventrolateral medulla oblongata. J Hypertens. v.17, n.9, p.1257-1263, 1999. 
MANCIA, G.; DAFFONCHIO, A.; DI RIENZO, M.; FERRARI, A.U.; GRASSI, G.

Methods to quantify sympathetic cardiovascular influences. Eur Heart J. v.19 Suppl F, p.F7-13, 1998.

MANCIA, G.; GRASSI, G.; GIANNATTASIO, C.; SERAVALLE, G. Sympathetic Activation in the Pathogenesis of Hypertension and Progression of Organ Damage. Hypertension. v.34, p.724-728, 1999.

MORIMOTO, S.; SASAKI, S.; MIKI, S.; KAWA, T.; ITOH, H.; NAKATA, T.; TAKEDA, K.; NAKAGAWA, M. Pressor response to pulsatile compression of the rostral ventrolateral medulla mediated by nitric oxide and c-fos expression. Br J Pharmacol. v.129, n.5, p.859-864, 2000.

MORIMOTO, S.; SASAKI, S.; MIKI, S.; KAWA, T.; ITOH, H.; NAKATA, T.; TAKEDA, K.; NAKAGAWA, M.; NARUSE, S.; MAEDA, T. Pulsatile compression of the rostral ventrolateral medulla in hypertension. Hypertension. v.29, n.1 Pt 2, p.514-518, 1997.

MORIMOTO, S.; SASAKI, S.; TAKEDA, K.; FURUYA, S.; NARUSE, S.; MATSUMOTO, K.; HIGUCHI, T.; SAITO, M.; NAKAGAWA, M. Decreases in blood pressure and sympathetic nerve activity by microvascular decompression of the rostral ventrolateral medulla in essential hypertension. Stroke. v.30, n.8, p.1707-1710, 1999.

MORISE, T.; HORITA, M.; KITAGAWA, I.; SHINZATO, R.; HOSHIBA, Y.; MASUYA, H.; SUZUKI, M.; TAKEKOSHI, N. The potent role of increased sympathetic tone in pathogenesis of essential hypertension with neurovascular compression. J Hum Hypertens. v.14, n.12, p.807-811, 2000. 
NARAGHI, R.; GAAB，M.R.; WALTER，G.F.; KLEINEBERG，B. Arterial hypertension and neurovascular compression at the ventrolateral medulla. A comparative microanatomical and pathological study. J Neurosurg. v.77, n.1, p.103-112, 1992.

NARAGHI, R.; GEIGER, H.; CRNAC, J.; HUK, W.; FAHLBUSCH, R.; ENGELS, G.; LUFT, F.C. Posterior fossa neurovascular anomalies in essential hypertension. Lancet. v.344, n.8935, p.1466-1470, 1994.

NOLL, G.; WENZEL, R.R.; BINGGELI, C.; CORTI, C.; LUSCHER, T.F. Role of sympathetic nervous system in hypertension and effects of cardiovascular drugs. Eur Heart J. v.19 Suppl F, p.F32-38, 1998.

OPARIL, S.; CHEN, Y.G.; BERECEK, K.H.; CALHOUN, D.A.; WYSS, J.M. The Role of the Central Nervous System in Hypertension. In: Laragh, J.H. and Brenner, B.M., eds. Hypertension: Pathophysiology, Diagnosis, and Management. New York, NY: Raven Press. v.1, p.713-740, 1990.

PIERPAOLO, L.; MASTRONARDI, L.; STRANO, S.; DI BIASI, C.; TRASIMENI, G. Neurovascular conflict and essential arterial hypertension: MR evaluation. AJNR Am J Neuroradiol. v.17, n.1, p.195-196, 1996.

PlATANIA, N.; NICOLETTI, G.F.; BARBAGAllO, G.; ALBANESE, V. Concurrent trigeminal and glossopharyngeal neuralgia, hemifacial spasm and hypertension by neurovascular compression. Case report. J Neurosurg Sci. v.41, n.3, p.303-307, 1997.

ROSS, M.E. Pharmacodynamics. In: Hardman, J.G. and Limbird, L.E., eds. Godman and Gilman's: The Pharmacological Basis of Therapeutics. New York: McGraw-Hill. p.29-42, 1996. 
RUIZ, T.; SANCHÍS, M.D.; FIORAVANTE, A.M.; PIQUÉ, X.C. Some Predictors of Cardiovascular Mortality Among the Elderly Population of Botucatu (SP). Arq Bras Cardiol. v.77, n.4, p.349-354, 2001.

SAGLITZ, S.A.; GAAB, M.R. Investigations using magnetic resonance imaging: is neurovascular compression present in patients with essential hypertension? J Neurosurg. v.96, n.6, p.1006-1012, 2002.

SCHOBEL, H.P.; FRANK, H.; NARAGHI, R.; GEIGER, H.; TITZ, E.; HEUSSER, K. Hypertension in patients with neurovascular compression is associated with increased central sympathetic outflow. J Am Soc Nephrol. v.13, n.1, p.35-41, 2002.

SENDESKI, M.M.; CONSOLIM-COLOMBO, F.M.; RICCIO, G.M.; KRIEGER, E.M.G.; LEITE, C.C. Neurovascular compression and hypertension: evaluation through magnetic resonance imaging and proposal of a image based classification. J Hypertension. v.20, n.suppl.4, p.S132, 2002.

VALLBO, A.B., ET AL. Somatosensory, proprioceptive and sympathetic activity in human peripheral nerves. Physiol. Rev. v.59, p.919-957, 1979.

WALLIN, G.B.; ELAM, M. Microneurography and Autonomic Dysfunction. In: Low, P.A., eds. Clinical Autonomic Disorders. Rochester, Minnesota: Little, Brown and Company. p.243-252, 1993.

WATTERS, M.R.; BURTON, B.S.; TURNER, G.E.; CANNARD, K.R. MR screening for brain stem compression in hypertension. AJNR Am J Neuroradiol. v.17, n.2, p.217-221, 1996.

WILKINS, R.H. Neurovascular compression syndromes. Neurol Clin. v.3, n.2, p.359-372, 1985. 
WYSS, J.M.; CARLSON, S.H. The role of the central nervous system in hypertension. Curr Hypertens Rep. v.1, n.3, p.246-253, 1999. 